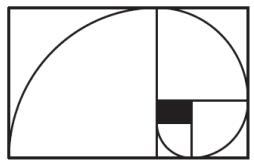

\title{
Braids and signatures
}

\section{Jean-Marc Gambaudo Étienne Ghys}

Originally published in Bulletin de la Societé Mathématique de France 133 (4), 2005, p. 541-579. Republication with permission from the Societé Mathématique de France.

\begin{abstract}
A braid defines a link which has therefore a well defined signature. This signature function defined on the braid group is not a homomorphism. Our goal is to describe the "defect of homomorphism" and to relate it to the Maslov cocycle associated to the classical Burau representation.
\end{abstract}




\section{Contents}

1 Introduction . . . . . . . . . . . . . 176

2 The Burau-Squier representation . . . . . . . . . 182

3 A visual computation . . . . . . . . . . . . . . 189

4 Braids with three strands . . . . . . . . . . . . . . 203

5 The gordian space . . . . . . . . . . . . . . . . . 210

Bibliography . . . . . . . . . . . . . . . . . . 214 


\section{Introduction}

This paper deals with the interaction of the following two standard constructions in knot theory.

Braids and Closed braids $[4,12]$

In the plane $\mathbf{R}^{2}$, we consider the sequence of points $x_{i}^{0}=(i, 0)$ (for $i=$ $1,2, \ldots)$ and we denote by $\mathbf{D}^{2}(r)$ the disc of radius $r$, centered at the origin. In the space $X_{n}$ of $n$-tuples of distinct points of $\mathbf{D}^{2}(n+1 / 2)$, we consider the equivalence relation that identifies two $n$-tuples if one is obtained from the other by a permutation of the indices. We denote by $\widetilde{X}_{n}$ the quotient space and by $\pi_{n}: X_{n} \rightarrow \widetilde{X}_{n}$ the natural projection. The fundamental group of $\widetilde{X}_{n}$, based at $\pi_{n}\left(x_{1}^{0}, x_{2}^{0}, \ldots, x_{n}^{0}\right)$, is called the $n$-th Artin braid group and is denoted by $\mathbf{B}_{n}$; its elements are called braids. Any braid $\gamma$ in $\mathbf{B}_{n}$ is represented by a path $t \in[0,1] \mapsto\left(x_{1}^{t}, x_{2}^{t}, \ldots, x_{n}^{t}\right) \in X_{n}$ i.e. by a system of $n$ disjoint arcs $t \mapsto\left(t, x_{i}^{t}\right)$ in the cylinder $[0,1] \times \mathbf{D}^{2}(n+1 / 2)$, such that $\pi_{n}\left(x_{1}^{1}, x_{2}^{1}, \ldots, x_{n}^{1}\right)=\pi_{n}\left(x_{1}^{0}, x_{2}^{0}, \ldots, x_{n}^{0}\right)$.

The identification $(x, 0) \approx(x, 1)$ for all $x$ in $\mathbf{D}^{2}(n+1 / 2)$ produces a finite collection of simple closed oriented curves in the solid torus $\mathbf{R} / \mathbf{Z} \times \mathbf{D}^{2}(n+1 / 2)$, images of the arcs $t \mapsto\left(t, x_{i}^{t}\right)$. The usual embedding of the solid torus in 3-space $\mathbf{R}^{3}$ and the compactification of $\mathbf{R}^{3}$ with a point at infinity, allow us to associate with any braid $\gamma$ an oriented link i.e. a collection of disjoint embeddings of an oriented circle in the 3 -sphere $\mathbf{S}^{3}$, called the closed braid associated with $\gamma$, and denoted by $\hat{\gamma}$ (see Figure 1 ).

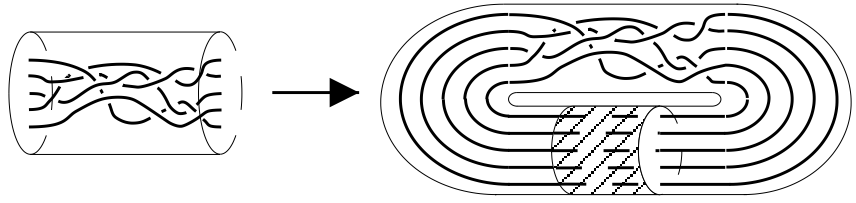

Figure 1: Closure of a braid

Signature of links $[7,11]$

Let $\lambda \subset \mathbf{S}^{3}$ be an oriented link in $\mathbf{S}^{3}$ and let us choose a Seifert surface: an oriented surface $S_{\lambda}$ embedded in $\mathbf{S}^{3}$ whose oriented boundary is $\lambda$. The first homology group $H_{1}\left(S_{\lambda} ; \mathbf{Z}\right)$ is equipped with a bilinear Seifert form $B$ in the following way. If $x$ and $y$ are two oriented closed curves on $S_{\lambda}$, one defines $B(x, y)$ as the linking number of $x$ with a curve $y^{\star}$ obtained from $y$ by pushing $y$ a little away from $S_{\lambda}$ along the positive direction transverse to $S_{\lambda}$. Clearly $B(x, y)$ only depends on the homology classes of $x$ and $y$ on 
$S_{\lambda}$. Turning $B$ into a symmetric bilinear form $\tilde{B}(x, y)=B(x, y)+B(y, x)$ and tensoring by $\mathbf{R}$, we get a symmetric bilinear form on the vector space $H_{1}\left(S_{\lambda} ; \mathbf{R}\right)$. It turns out that the signature of this symmetric form is independent of the choice of the Seifert surface: it is the signature of the oriented link $\lambda$, denoted $\operatorname{sign}(\lambda) \in \mathbf{Z}$. For definiteness, we recall that the signature of a quadratic form is the number of + signs minus the number of - signs in an orthogonal basis.

The notion of signature of an oriented link can be generalized as follows. Tensoring by $\mathbf{C}$, we get a bilinear form on the vector space $H_{1}\left(S_{\lambda} ; \mathbf{C}\right)$. Consider a complex number $\omega \neq 1$ (usually chosen as a root of unity) and the hermitian form $\tilde{B}_{\omega}(x, y)=(1-\omega) B(x, \bar{y})+(1-\bar{\omega}) B(\bar{y}, x)$. The signature of this hermitian form is again independent of the choice of the Seifert surface: it is the $\omega$-signature, $\operatorname{sign}_{\omega}(\lambda) \in \mathbf{Z}$, of the oriented link $\lambda$. In the case $\omega=-1$, we recover the signature of the oriented link.

There is a natural sequence of embeddings of the braid groups $\mathbf{B}_{1} \subset$ $\mathbf{B}_{2} \subset \ldots \subset \mathbf{B}_{n} \subset \ldots$ The embedding $i_{n}$ of $\mathbf{B}_{n}$ in $\mathbf{B}_{n+1}$ amounts to adding an additional "trivial" strand (see Figure 2). The union of this infinite chain of groups is the infinite braid group $\mathbf{B}_{\infty}$. Note that $\operatorname{sign}_{\omega}(\alpha)=\operatorname{sign}_{\omega}\left(i_{n}(\alpha)\right)$ since a Seifert surface for $\widehat{i_{n}(\alpha)}$ is obtained from a Seifert surface for $\hat{\alpha}$ by adding a disjoint disc. Therefore, the function $\operatorname{sign}_{\omega}(\hat{\alpha})$ is well defined on $\mathbf{B}_{\infty}$.

Combining these constructions, for each $\omega$, we get a map from $\mathbf{B}_{\infty}$ to $\mathbf{Z}$ which associates with a braid $\gamma$ the signature $\operatorname{sign}_{\omega}(\hat{\gamma})$. We are now in a position to raise the question:

Given two braids $\alpha$ and $\beta$ in the braid group $\mathbf{B}_{\infty}$ and a root of unity $\omega$, what can be said about the quantity:

$$
\operatorname{sign}_{\omega}(\widehat{\alpha \cdot \beta})-\operatorname{sign}_{\omega}(\hat{\alpha})-\operatorname{sign}_{\omega}(\hat{\beta}) ?
$$

In this paper we give an explicit answer to this question. Before giving a precise statement we need to recall another construction which again is standard in low dimensional topology.

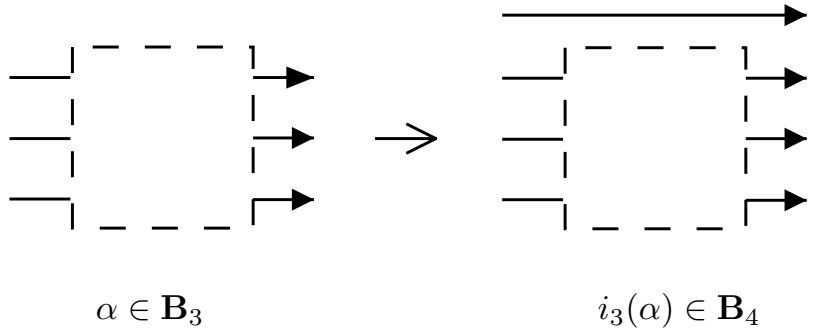

Figure 2: Adding a trivial strand 
The Burau representation and the Meyer Cocycle

Burau defined an explicit linear representation of $\mathbf{B}_{n}$ in $\mathbf{G L}(n-$ $\left.1, \mathbf{Z}\left[t, t^{-1}\right]\right)$, where $t$ denotes some indeterminate. These representations combine to a representation of $\mathbf{B}_{\infty}$ in the ascending union $\mathbf{G} \mathbf{L}\left(\infty, \mathbf{Z}\left[t, t^{-1}\right]\right)$ of the $\mathbf{G L}\left(n-1, \mathbf{Z}\left[t, t^{-1}\right]\right)$.

If one specializes $t$ as a complex number $\omega$, we get a linear representation $\mathcal{B}_{\omega}$ in $\mathbf{G L}(\infty, \mathbf{C})$. In [13], Squier shows that if $\omega$ is a complex number of modulus 1 , the image of $\mathcal{B}_{\omega}$ is contained in the unitary group of some non degenerate hermitian form. Since the imaginary part of such a hermitian form is a symplectic form, the Burau representation provides symplectic representations:

$$
\mathcal{B}_{\omega}: \mathbf{B}_{\infty} \rightarrow \mathbf{S p}(\infty, \mathbf{R})
$$

where we denote by $\mathbf{S p}(\infty, \mathbf{R})$ the ascending union of the symplectic groups $\mathbf{S p}(2 g, \mathbf{R})$ (consisting of the symplectic automorphisms $\gamma$ of $\mathbf{R}^{\infty}$ which are the identity on all vectors of the canonical basis of $\mathbf{R}^{\infty}$ except for a finite number of them).

In Section 2, we shall give more information concerning this BurauSquier representation. We shall give explicit formulas for the symplectic form and a topological interpretation which (hopefully) will shed some light on the symplectic nature of the Burau representation, originally introduced by Squier in purely algebraic terms.

The symplectic group $\mathbf{S p}(2 g, \mathbf{R})$ is not simply connected; its universal cover $\widetilde{\mathbf{S p}}(2 g, \mathbf{R})$ defines a central extension

$$
0 \rightarrow \mathbf{Z} \rightarrow \widetilde{\mathbf{S p}}(2 g, \mathbf{R}) \rightarrow \mathbf{S p}(2 g, \mathbf{R}) \rightarrow 1 .
$$

This determines a cohomology class in $H^{2}(\mathbf{S p}(2 g, \mathbf{R}) ; \mathbf{Z})$, called the Maslov class. It turns out that 4 times the Maslov class can be represented explicitly by an integral valued Meyer cocycle which is invariant by conjugation. We shall give more motivation and background for this cocycle in Subsection 3.2 but for the time being we only mention the following "computational" definition (see [9]):

Let $\gamma_{1}$ and $\gamma_{2}$ be two elements in $\mathbf{S p}(2 g, \mathbf{R})$ and denote by $E_{\gamma_{1}, \gamma_{2}}$ the intersection of the images of $\gamma_{1}^{-1}-i d$ and $\gamma_{2}-i d$. If $e$ belongs to $E_{\gamma_{1}, \gamma_{2}}$, choose two vectors $v_{1}$ and $v_{2}$ such that

$$
e=\gamma_{1}^{-1}\left(v_{1}\right)-v_{1}=v_{2}-\gamma_{2}\left(v_{2}\right)
$$

and define

$$
q_{\gamma_{1}, \gamma_{2}}(e)=\Omega\left(v_{1}+v_{2}, e\right)
$$

where $\Omega$ denotes the standard symplectic form on $\mathbf{R}^{2 g}$. One checks easily that $q_{\gamma_{1}, \gamma_{2}}(e)$ is independent of the choices of $v_{1}$ and $v_{2}$ and defines a quadratic form on $E_{\gamma_{1}, \gamma_{2}}$. By definition, the evaluation of the Meyer cocycle on the pair $\left(\gamma_{1}, \gamma_{2}\right)$, denoted $\operatorname{Meyer}\left(\gamma_{1}, \gamma_{2}\right)$, is the signature of 
this quadratic form. Observe that the Meyer cocycle can be coherently defined for elements in $\mathbf{S p}(\infty, \mathbf{R})$. In other words, if $\gamma_{1}$ and $\gamma_{2}$ are two elements in $\mathbf{S p}(2 g, \mathbf{R})$ seen as elements $\gamma_{1}^{\prime}$ and $\gamma_{2}^{\prime}$ of $\mathbf{S p}(2 g+2, \mathbf{R})$, the values of $\operatorname{Meyer}\left(\gamma_{1}, \gamma_{2}\right)$ and $\operatorname{Meyer}\left(\gamma_{1}^{\prime}, \gamma_{2}^{\prime}\right)$ coincide.

We hope that this mysterious definition will become crystal clear in Section 3.2.

We now state the main result of this paper:

Theorem A: Let $\alpha$ and $\beta$ two braids in $\mathbf{B}_{\infty}$ and $\omega \neq 1$ a root of unity. Then:

$$
\operatorname{sign}_{\omega}(\widehat{\alpha \cdot \beta})-\operatorname{sign}_{\omega}(\hat{\alpha})-\operatorname{sign}_{\omega}(\hat{\beta})=-\operatorname{Meyer}\left(\mathcal{B}_{\omega}(\alpha), \mathcal{B}_{\omega}(\beta)\right) .
$$

Remark 1: Since the Meyer cocycle evaluated on $\mathbf{S p}(2 g, \mathbf{R})$ is the signature of a quadratic form on a vector space with dimension smaller that $2 g$, it follows easily from the definition of the Burau-Squier representation that, for any positive integer $n$, and any pair of braids $\alpha$ and $\beta$ in $\mathbf{B}_{n}$, we have:

$$
\left|\operatorname{sign}_{\omega}(\widehat{\alpha \cdot \beta})-\operatorname{sign}_{\omega}(\hat{\alpha})-\operatorname{sign}_{\omega}(\hat{\beta})\right| \leq 2 n \text {. }
$$

Thus, for any positive integer $n$, the map $\alpha \in \mathbf{B}_{n} \mapsto \operatorname{sign}_{\omega}(\hat{\beta}) \in \mathbf{R}$ is a quasimorphism. A direct proof of this result can be found in [5] where the authors use this property to construct non trivial quasimorphisms on the group of area preserving diffeomorphisms of the 2 -sphere.

Remark 2: The Artin braid group $\mathbf{B}_{n}$ has a standard presentation in terms of generators $\sigma_{1}, \sigma_{2}, \ldots, \sigma_{n-1}$ and relations:

$$
\begin{gathered}
\sigma_{i} \cdot \sigma_{j}=\sigma_{j} \cdot \sigma_{i} \\
\sigma_{i} \cdot \sigma_{i+1} \cdot \sigma_{i}=\sigma_{i+1} \cdot \sigma_{i} \cdot \sigma_{i+1}
\end{gathered}
$$

for all $i, j$ in $\{1, \ldots, n-1\}$ satisfying $|i-j| \geq 2$. See for instance [11].

The closed braids $\widehat{\sigma}_{i}$ are trivial links. Given a braid $\beta$ in $\mathbf{B}_{n}$ which reads $\beta=\sigma_{i_{1}} \cdots \sigma_{i_{l}}$, we have:

$$
\operatorname{sign}_{\omega}(\hat{\beta})=-\sum_{j=2}^{j=l} \operatorname{Meyer}\left(\mathcal{B}_{\omega}\left(\sigma_{i_{1}} \cdots \sigma_{i_{l-1}}\right), \mathcal{B}_{\omega}\left(\sigma_{i_{l}}\right)\right) .
$$

This last formula is actually very easy to use for numerical computations since the matrices $\mathcal{B}_{\omega}\left(\sigma_{i_{l}}\right)$ are sparse (as we shall see in Section 2).

Remark 3: As a trivial illustration of Theorem A, consider the case $n=2$. The image of $\mathcal{B}_{-1}\left(\mathbf{B}_{2}\right)$ is contained in $\mathbf{S p}(2, \mathbf{R})=\mathbf{S L}(2, \mathbf{R})$ and it is not 
difficult to see that, up to conjugacy, $\mathcal{B}_{-1}\left(\sigma_{1}\right)$ is the matrix $\left(\begin{array}{cc}1 & 0 \\ -1 & 1\end{array}\right)$. Evaluating the Meyer cocycle on $2 \times 2$ unipotent matrices is very easy so that one can compute $\operatorname{sign}\left(\sigma_{1}^{l}\right)$ using the method explained in Remark 2. The reader will find immediately the value $1-l$ for $l \geq 1$. Of course, one can also compute this signature using an explicit Seifert surface for this elementary braid (see [11]).

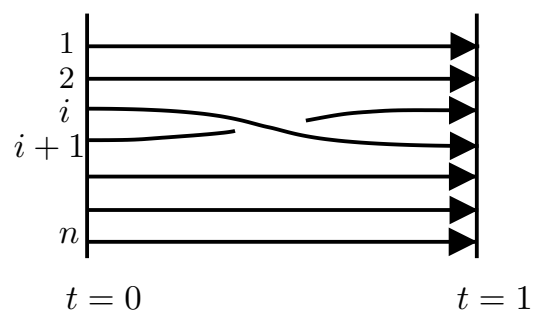

Figure 3: The braid $\sigma_{i}$

Section 3 is devoted to the proof of Theorem A. This proof will be a visual computation (following an expression borrowed from [14]). First, we interpret both the signature of an oriented link and the evaluation of the Meyer cocycle as the signatures of the intersection forms of some 4manifolds (subsections 3.1 and 3.2). Then, in 3.3 we construct an oriented compact connected 4-manifold $M_{2}^{4}(\alpha, \beta)$ which is a 2-fold branched cover of $S_{2} \times \mathbf{D}^{2}$ where $S_{2}$ is the oriented surface with genus 2 and the branching locus is a suitable closed surface. We shall compute the signature $\sigma\left(M_{2}^{4}(\alpha, \beta)\right)$ of the intersection form on $M_{2}^{4}(\alpha, \beta)$ in two different ways; these computations will give us the equality stated in Theorem $\mathrm{A}$ in the case $\omega=-1$. The proof of Theorem $\mathrm{A}$ in the case of a general root of unity is given in 3.4: it is an elaboration of the previous proof in a setting which is equivariant under some finite cyclic group.

In Sections 4 and 5, we give two simple applications of these results.

The first is an explicit computation of the signature on the braid group with three strands $\mathbf{B}_{3}$. Denote by $l k_{3}: \mathbf{B}_{3} \rightarrow \mathbf{Z}$ the (linking) homomorphism mapping each generator to 1 . Recall that the center of $\mathbf{B}_{3}$ is generated by the element $\Delta_{3}^{2}=\left(\sigma_{1} \cdot \sigma_{2} \cdot \sigma_{1}\right)^{2}$ and that the quotient of $\mathbf{B}_{3}$ by the group generated by $\Delta_{3}^{4}$ is isomorphic to $\mathbf{S L}(2, \mathbf{Z})$. In [1], Atiyah defines many functions from $\mathbf{S L}(2, \mathbf{Z})$ to $\mathbf{Z}$ of different origins (topological, analytical, and number theoretical) and proves that they all essentially coincide. We refer to [3] for another approach to this coincidence. One of the versions of these functions on $\mathbf{S L}(2, \mathbf{Z})$ is called in [3] the Rademacher function (we recall a definition in Section 4.2). The following theorem adds 
a new element to this long list of (identical!) functions on $\mathbf{S L}(2, \mathbf{Z}) \ldots$ It has been announced (and used!) in an earlier paper of the authors [5].

Theorem B: The function sign $+\frac{2}{3} l k_{3}$ descends to a function on $\mathbf{S L}(2, \mathbf{Z})$ which coincides with $-1 / 3$ times the Rademacher function.

Finally, we show how this knowledge of signatures gives some information concerning the rough geometry of the gordian distance. Denote by $\mathcal{K}$ nots the set of (isotopy classes of) knots in 3 -space. There is a natural distance $d_{\text {gordian }}$ on $\mathcal{K}$ nots that we now define. Given two knots $f_{0}, f_{1}: \mathbf{S}^{1} \hookrightarrow \mathbf{R}^{3}$, one considers homotopies $\left(f_{t}\right)_{t \in[0,1]}: \mathbf{S}^{1} \rightarrow \mathbf{R}^{3}$ which connect the two knots and are such that for each $t \in[0,1]$, the curve $f_{t}$ is an immersion which has at most one double point, this double point being generic (the two local arcs that intersect have distinct tangents at the intersection). Denote by $D\left(\left(f_{t}\right)_{t \in[0,1]}\right)$ the total number of double points of this family of curves. The gordian distance between the two knots $f_{0}$ and $f_{1}$ is the minimum of $D\left(\left(f_{t}\right)_{t \in[0,1]}\right)$ for all such homotopies connecting the knots.

The global geometry of this (discrete) metric space is quite intriguing and probably very intricate. Note for instance that this space is not locally finite (an infinite number of knots can be made trivial by allowing one crossing as the reader will check on the examples given on Figure 4, which can be easily distinguished by their Alexander polynomials). As a first approach, we propose to study the coarse geometry of this metric space, i.e. up to quasi-isometries. Based on the observation that the signatures give lower bounds for the gordian distance (see [15]) we prove that the gordian metric space contains "quasi-euclidean subspaces" of arbitrary dimensions.

Theorem C: For every integer $d \geq 1$, there is a map $\xi: \mathbf{Z}^{d} \rightarrow \mathcal{K}$ nots which is a quasi-isometry onto its image, i.e. such that the gordian distance between $\xi(x)$ and $\xi(y)$ satisfies

$$
A\|x-y\|-B \leq d_{\text {gordian }}(\xi(x), \xi(y)) \leq C\|x-y\|
$$

for some constants $A, B, C>0$ and some norm $\|\cdot\|$ in $\mathbf{R}^{d}$.

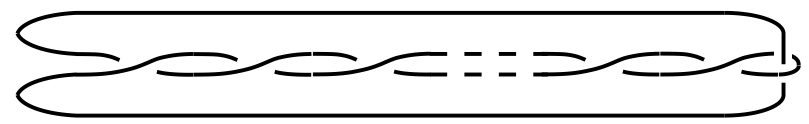

Figure 4: Knots at distance 1 from the trivial knot

It would be interesting to find quasi-isometric embeddings of other nice metric spaces, like for instance an infinite regular tree. Another puzzling 
question concerning this space is to know whether or not it admits non trivial global quasi-isometries, i.e. bijections of $\mathcal{K}$ nots which quasi-preserve the gordian distance without being at a bounded distance from the identity (or from the involutions reversing orientations of the knot, of ambient space, or both). We could also ask for the space of "ends": connect two points in $\mathcal{K}$ nots by an edge if their gordian distance is 1 and consider unbounded connected components of the complements of large balls in this graph.

All along this paper, we shall say that the orientation of an oriented manifold $M$ is compatible with the orientation of one of its boundary components $N$ if a positive basis of the tangent space to $N$ followed with a normal vector to $N$ pointing inward $M$ is a positive basis of the tangent space to $M$. Several other conventions are in order in this paper and we hope that we used them consistently!

We would like to thank the referee for his/her constructive comments which helped us to improve the presentation of this paper.

\section{The Burau-Squier representation}

Most of this section is probably well known to experts and is included here for the convenience of the reader and because it is necessary for the sequel of this paper. We would like to interpret the classical Burau representation in terms of the action of the braid groups on the homology of some branched covers of the disc. This will also provide explicit formulas for all these representations. We shall partly follow a presentation of Kolev [8].

\subsection{The Burau representation}

Consider the homomorphism $\phi_{n}$ from the fundamental group of the punctured disc $\mathbf{D}^{2} \backslash\left\{x_{1}^{0}, \ldots, x_{n}^{0}\right\}$ to $\mathbf{Z}$ which maps every (conjugacy class of) small positive simple loop around each base point $x_{i}^{0}$ to the generator 1 in $\mathbf{Z}$. The kernel of this homomorphism defines an infinite cyclic covering $D_{n}^{\infty}$ of $\mathbf{D}^{2} \backslash\left\{x_{1}^{0}, \ldots, x_{n}^{0}\right\}$. Figure 5 illustrates this covering for $n=3$ and shows the graph of $z \mapsto \arg \left(z^{3}-1\right)$.

A standard result by Birman [4] states that the Artin braid group $\mathbf{B}_{n}$ is isomorphic to the group of isotopy classes of homeomorphisms of the disc $\mathbf{D}^{2}(n+1 / 2)$ that fix the boundary $\partial \mathbf{D}^{2}(n+1 / 2)$ pointwise and leave globally invariant the set of points $\left\{x_{1}^{0}, \ldots, x_{n}^{0}\right\}$. Thus, each braid $\beta$ in $\mathbf{B}_{n}$ can be represented by some homeomorphism $h_{\beta}$ of $\mathbf{D}^{2}(n+1 / 2)$. We choose the lift $\tilde{h}_{\beta}$ of $h_{\beta}$ to $D_{n}^{\infty}$ that leaves the boundary pointwise fixed. Clearly this lift is not uniquely defined by $\beta$ but its homotopy class is. In particular, the map $\left(\tilde{h_{\beta}}\right)_{\star}$ induced by $\tilde{h}_{\beta}$ on the first homology group of $D_{n}^{\infty}$ is determined by $\beta$. Note that the braid $\alpha \cdot \beta$ consists of $\alpha$ followed by 


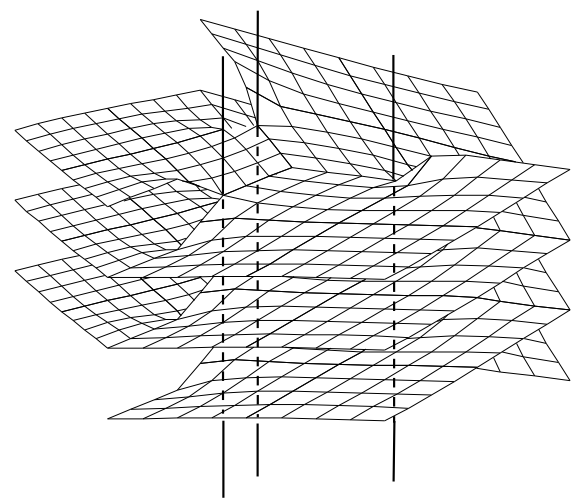

Figure 5: Covering of the punctured disc

$\beta$ so that we get a linear anti-representation (rather than a representation) of $\mathbf{B}_{n}$ in the group of automorphisms of $H_{1}\left(D_{n}^{\infty} ; \mathbf{R}\right)$. If we consider the dual action on the first cohomology with compact support, we get a linear representation of $\mathbf{B}_{n}$.

In order to produce an explicit formula for this representation, we choose a base point $z$ in $\mathbf{D}^{2} \backslash\left\{x_{1}^{0}, \ldots, x_{n}^{0}\right\}$ and embed a wedge $R_{n}$ of $n$ circles based at $z$, made of $n$ positive simple loops $e_{1}, \ldots, e_{n}$ going once around $x_{1}^{0}, \ldots, x_{n}^{0}$ respectively. Choose some lift $\tilde{z}$ of $z$ in $D_{n}^{\infty}$ and denote by $\tilde{e}_{1}, \ldots, \tilde{e}_{n}$ the paths lifting $e_{1}, \ldots, e_{n}$ and starting from $\tilde{z}$. The inverse image of $R_{n}$ in $D_{n}^{\infty}$ is a 1-complex $\tilde{R}_{n}^{\infty}$ and we denote by $\tilde{C}_{n}^{\infty}$ the module of integral 1-chains on $\tilde{R}_{n}^{\infty}$, which can be seen as a $\mathbf{Z}\left[t, t^{-1}\right]$-module, using the deck transformations. This is a free module on $\tilde{e}_{1}, \ldots, \tilde{e}_{n}$. The submodule $\tilde{Z}_{n}^{\infty}$ of 1 -cycles consists of those chains in $\tilde{R}_{n}^{\infty}$ such that the sum of the coordinates in this basis is zero. Therefore, $\tilde{Z}_{n}^{\infty}$ is the free module on $v_{1}=\tilde{e}_{1}-\tilde{e}_{2} ; v_{2}=\tilde{e}_{2}-\tilde{e}_{3} ; \ldots ; v_{n-1}=\tilde{e}_{n-1}-\tilde{e}_{n}$.

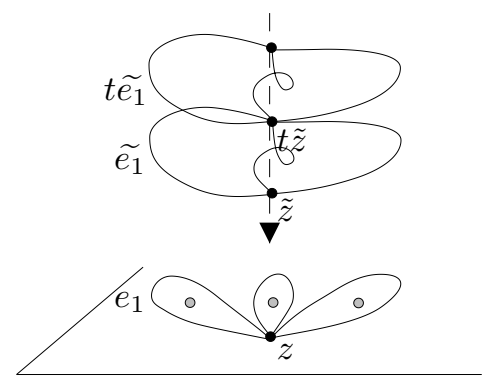

Figure 6: The complex $R_{n}$ and its cover 
The action of any braid in $\mathbf{B}_{n}$ on the first homology of $D_{n}^{\infty}$ commutes with the action of deck transformations. This homology is isomorphic to $\tilde{Z}_{\infty}$ as a $\mathbf{Z}\left[t, t^{-1}\right]$-module. By definition, the dual action on cohomology with compact support, in the dual basis, is the (reduced) Burau representation $\mathcal{B}_{t}$. In the standard presentation of $\mathbf{B}_{n}$, the images of the generators $\sigma_{i}$ are the matrices

$$
\begin{aligned}
\mathcal{B}_{t}\left(\sigma_{1}\right)=\left(\begin{array}{ccccc}
-t^{-1} & 0 & 0 & \ldots & 0 \\
t^{-1} & 1 & 0 & \ldots & 0 \\
0 & 0 & 1 & & 0 \\
\vdots & \vdots & & \ddots & \\
0 & 0 & 0 & \ldots & 1
\end{array}\right), \mathcal{B}_{t}\left(\sigma_{n-1}\right)=\left(\begin{array}{ccccc}
1 & & 0 & 0 & 0 \\
& \ddots & & \vdots & \vdots \\
0 & \ldots & 1 & 0 & 0 \\
0 & 0 & 0 & 1 & 1 \\
0 & 0 & 0 & 0 & -t^{-1}
\end{array}\right), \\
\mathcal{B}_{t}\left(\sigma_{i}\right)=\left(\begin{array}{ccccccc}
1 & \ldots & 0 & 0 & 0 & \ldots & 0 \\
& \ddots & & & & & \vdots \\
0 & & 1 & 1 & 0 & & 0 \\
0 & & 0 & -t^{-1} & 0 & & 0 \\
0 & & 0 & t^{-1} & 1 & & 0 \\
\vdots & & & & & \ddots & \vdots \\
0 & \ldots & 0 & 0 & 0 & \ldots & 1
\end{array}\right)
\end{aligned}
$$

(for $2 \leq i \leq n-2$, where the diagonal $-t^{-1}$ is in position $(i, i)$ ).

We also denote by $\mathcal{B}_{t}$ the corresponding linear representation of $\mathbf{B}_{\infty}$ in the ascending union $\mathbf{G L}\left(\infty, \mathbf{Z}\left[t, t^{-1}\right]\right)$ of the $\mathbf{G L}\left(n-1, \mathbf{Z}\left[t, t^{-1}\right]\right)$.

Remark 1: The convention about the choice of the generators of the braid group is not uniform in the literature. Many authors use the generators $\sigma_{i}^{\prime}=\sigma_{i}^{-1}$. This remark will help the reader to convince himself or herself that the above Burau representation is indeed the Burau representation given in [13].

Remark 2: The matrices that one gets using the action in homology are obviously transposed of the previous ones. When referring to these matrices, we will speak about the Burau anti-representation and transposed Burau matrices.

\subsection{Branched covers of the disc}

The 2 -fold cover of the disc $\mathbf{D}^{2}(n+1 / 2)$ branched at the points $x_{1}^{0}, \ldots, x_{n}^{0}$ is an oriented surface with genus $(n-1) / 2$ (resp. $n / 2-1)$ and one (resp. two) boundary component(s) when $n$ is odd (resp. even). We denote this surface by $F_{n, 2}$. All the surfaces $F_{n, 2}$ can be naturally nested and we denote by $F_{\infty, 2}$ the union of these compact surfaces with boundary. See Figure 7. 


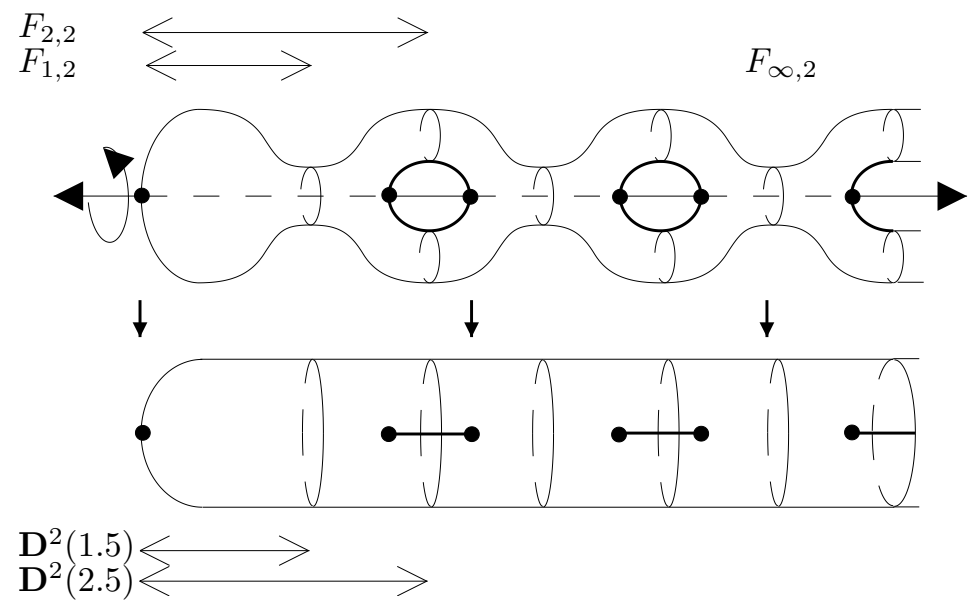

Figure 7: The surface $F_{\infty, 2}$ ramified over the plane

The cohomology group $H^{1}\left(F_{n, 2}, \partial F_{n, 2} ; \mathbf{R}\right)$ is a vector space of dimension $n-1$ which embeds in the cohomology group $H_{c}^{1}\left(F_{\infty, 2} ; \mathbf{R}\right) \simeq \mathbf{R}^{\infty}$ with compact support.

Each braid $\beta$ in $\mathbf{B}_{n}$ can be represented by some homeomorphism $h_{\beta}$ of $\mathbf{D}^{2}(n+1 / 2)$. We choose the lift $\tilde{h}_{\beta}$ of $h_{\beta}$ to $F_{n, 2}$ that leaves the boundary component(s) of $F_{n, 2}$ pointwise fixed. Clearly the homotopy class of this lift is uniquely determined. In particular, the map $\tilde{h}_{\beta}^{\star}$ induced by $\tilde{h}_{\beta}$ on the first relative cohomology group $H^{1}\left(F_{n, 2}, \partial F_{n, 2} ; \mathbf{R}\right)$ is determined by $\beta$ and produces a representation of $\mathbf{B}_{n}$ as a group of automorphisms of $H^{1}\left(F_{n, 2}, \partial F_{n, 2} ; \mathbf{R}\right)$.

In the next subsection, we shall prove the following proposition which gives a topological interpretation of the Burau representation $\mathcal{B}_{t}$ evaluated at $t=-1$ (which is seen as a representation $\mathcal{B}_{-1}$ from $\mathbf{B}_{n}$ in $\mathbf{G L}(n-1, \mathbf{R})$ ).

Proposition 2.1. There is an isomorphism between $H^{1}\left(F_{n, 2}, \partial F_{n, 2} ; \mathbf{R}\right)$ and $\mathbf{R}^{n-1}$ which conjugates the representations of the braid group $\mathbf{B}_{n}$ given by $\tilde{h}_{\beta}^{\star}$ and by $\mathcal{B}_{-1}$.

Similarly, one can interpret the Burau representation evaluated at roots of unity. We fix some integer $k \geq 3$.

Denote by $F_{n, k}$ the cyclic cover of order $k$ of $\mathbf{D}^{2}$ branched over the base points $x_{1}^{0}, \ldots, x_{n}^{0}$. To be more precise, consider the homomorphism from the fundamental group of $\mathbf{D}^{2} \backslash\left\{x_{1}^{0}, \ldots, x_{n}^{0}\right\}$ to $\mathbf{Z} / k \mathbf{Z}$ which maps every (conjugacy class of) small positive simple loop around each base point $x_{i}^{0}$ to the generator 1 in $\mathbf{Z} / k \mathbf{Z}$. The kernel of this homomorphism defines a regular $k$-fold cover over $\mathbf{D}^{2} \backslash\left\{x_{1}^{0}, \ldots, x_{n}^{0}\right\}$ which in turn defines a branched cover $F_{n, k}$ over the disc. The surface $F_{n, k}$ has $\operatorname{gcd}(n, k)$ components in its boundary. The braid group $\mathbf{B}_{n}$ acts linearly on the cohomology group 
$H^{1}\left(F_{n, k}, \partial F_{n, k} ; \mathbf{R}\right)$. Note that there is a natural action of $\mathbf{Z} / k \mathbf{Z}$ on $F_{n, k}$ and the induced action on $H^{1}\left(F_{n, k}, \partial F_{n, k} ; \mathbf{R}\right)$ commutes with this linear representation of $\mathbf{B}_{n}$. The surfaces $F_{n, k}$ for a fixed $k$ are naturally nested and we denote by $F_{\infty, k}$ the union of these compact surfaces. As before, these linear representations can be unified in a linear representation of $\mathbf{B}_{\infty}$ on $H_{c}^{1}\left(F_{\infty, k} ; \mathbf{R}\right) \simeq \mathbf{R}^{\infty}$.

Any complex vector space $E$ equipped with a linear representation of $\mathbf{Z} / k \mathbf{Z}$ can be decomposed in eigenspaces $E=\bigoplus_{\omega^{k}=1} E_{\omega}$ in such a way that $1 \in \mathbf{Z} / k \mathbf{Z}$ acts on $E_{\omega}$ by multiplication by $\omega$. This decomposition is invariant under any complex linear map commuting with the $\mathbf{Z} / k \mathbf{Z}$ action. If the vector space $E$ is a real vector space, this applies to the complexification $E \otimes \mathbf{C}$ and eigenspaces corresponding to conjugate eigenvalues are conjugate subspaces. This gives an invariant "eigenspace decomposition" of $E$ as a sum of real vector spaces $E_{\omega}$ where $E_{\omega}=E_{\bar{\omega}}$. The action of the generator $1 \in \mathbf{Z} / k \mathbf{Z}$ on $E_{\omega}$ is a linear map which is $i d$ if $\omega=1,-i d$ if $\omega=-1$, and is conjugate to a matrix consisting of $2 \times 2$ diagonal blocks, each being a rotation by the argument of $\omega$ otherwise.

Applying these remarks to the vector space $H^{1}\left(F_{n, k}, \partial F_{n, k} ; \mathbf{R}\right)$ we get subspaces $H^{1}\left(F_{n, k}, \partial F_{n, k} ; \mathbf{R}\right)_{\omega}$ equipped with linear actions of the braid group $\mathbf{B}_{n}$. We can now state the topological interpretation of $\mathcal{B}_{\omega}$ acting in $\mathbf{C}^{n-1}$ that we prove in the next subsection.

Proposition 2.2. There is an isomorphism between $H^{1}\left(F_{n, k}, \partial F_{n, k} ; \mathbf{R}\right)_{\omega}$ and $\mathbf{R}^{2 n-2} \simeq \mathbf{C}^{n-1}$ which conjugates the representations of the braid group $\mathbf{B}_{n}$ given by $\tilde{h}_{\beta}^{\star}$ and by $\mathcal{B}_{\omega}$.

\subsection{The Squier representation}

We first prove propositions 2.1 and 2.2. One can compute the homology of the $k$-fold (unramified) cover $D_{n}^{k}$ of $\mathbf{D}^{2} \backslash\left\{x_{1}^{0}, \ldots, x_{n}^{0}\right\}$ associated to the reduction of the homomorphism $\phi_{n}$ modulo $k$. Denote by $\tilde{C}_{n}^{k}$ the module of 1-chains on the inverse image $\tilde{R}_{n}^{k}$ of $R_{n}$ in $D_{n}^{k}$. This can be seen as a free $\mathbf{Z}\left[t, t^{-1}\right] /\left(t^{k}-1\right.$ )-module on $\tilde{e}_{1}^{k}, \ldots, \tilde{e}_{n}^{k}$ (where the $\tilde{e}_{i}^{k}$ denote the lifts of $e_{i}$ to $D_{n}^{k}$ starting from a base point $\tilde{z}$ ). The sub-module $\tilde{Z}_{n}^{k}$ of 1-cycles consists of those chains in $\tilde{R}_{n}^{k}$ such that the sum of the coordinates in this basis is a constant multiple of $\left(1+t+\ldots+t^{k-1}\right)$. Therefore, $\tilde{Z}_{n}^{k}$ is the sum of a free module on $v_{1}^{k}=\tilde{e}_{1}^{k}-\tilde{e}_{2}^{k} ; v_{2}^{k}=\tilde{e}_{2}^{k}-\tilde{e}_{3}^{k} ; \ldots ; v_{n-1}^{k}=\tilde{e}_{n-1}^{k}-\tilde{e}_{n}^{k}$ and the module generated by $\left(1+t+\ldots+t^{k-1}\right)\left(\tilde{e}_{1}^{k}\right)$ (on which the action of $t$ is trivial).

From the knowledge of the homology of the cover $D_{n}^{k}$, one deduces the homology of the branched cover $F_{n, k}$ over $\mathbf{D}^{2}$. It is enough to add the additional relation $1+t+\ldots+t^{k-1}=0$ which is clear: the $k$-th power of the loops $e_{i}$ lift to loops which bound discs in $F_{n, k}$. It follows that the homology of $F_{n, k}$ is isomorphic to the free $\mathbf{Z}\left[t, t^{-1}\right] /\left(1+t+\ldots+t^{k-1}\right)$ module on $v_{1}^{k}=\tilde{e}_{1}^{k}-\tilde{e}_{2}^{k} ; v_{2}^{k}=\tilde{e}_{2}^{k}-\tilde{e}_{3}^{k} ; \ldots ; v_{n-1}^{k}=\tilde{e}_{n-1}^{k}-\tilde{e}_{n}^{k}$ (where 
we also denote by $\tilde{e}_{i}^{k}$ the lifts of the $e_{i}$ in $\left.F_{n, k}\right)$. This homology, as a $\mathbf{Z}$-module, is a free module of rank $(k-1)(n-1)$, isomorphic to $\mathbf{Z}^{n-1} \otimes\left(\mathbf{Z}\left[t, t^{-1}\right] /\left(1+t+\ldots+t^{k-1}\right)\right)$. The action of the braid group $\mathbf{B}_{n}$ is given by the Burau anti-representation where one adds the relation $1+t+\ldots+t^{k-1}=0$. The action of $\mathbf{Z} / k \mathbf{Z}$ is of course given by multiplication by $t$. If one tensors this by $\mathbf{C}$, it is easy to compute the eigenspace decomposition. The eigenspace corresponding to $\omega=1$ is trivial and the eigenspace corresponding to the root of unity $\omega \neq 1$ has dimension $n-1$ and is generated by

$$
w_{i}^{k}=\left(1+\bar{\omega} t+\bar{\omega}^{2} t^{2}+\ldots+\bar{\omega}^{k-1} t^{k-1}\right) \cdot v_{i}^{k} \quad(i=1, \ldots, n-1) .
$$

The action of the braid group on each of these eigenspaces, when written in the basis $w_{i}^{k}$, is precisely given by substituting $\omega$ to $t$ in the Burau anti-representation to produce a $(n-1) \times(n-1)$ complex matrix.

From all these considerations, it is easy to find the subspace associated to the eigenvalue $\omega$ in the real homology group $H_{1}\left(F_{n, k} ; \mathbf{R}\right)$ and the corresponding action of the braid group. If $\omega$ is not real (i.e. $\neq \pm 1$ ), then it is isomorphic to $\mathbf{C}^{n-1} \simeq \mathbf{R}^{2(n-1)}$, as a real vector space, and the action of $\mathbf{B}_{n}$ is given by the transposed Burau matrices evaluated at $\omega$, seen as real $(2 n-2) \times(2 n-2)$ matrices. When $\omega=-1$ the associated subspace in $H_{1}\left(F_{n, k} ; \mathbf{R}\right)$ is isomorphic to $\mathbf{R}^{n-1}$ and the action of $\mathbf{B}_{n}$ is given by the Burau matrices evaluated at -1 . Finally, the eigenspace corresponding to +1 is trivial.

This completes the proof of Propositions 2.1 and 2.2.

The cohomology groups $H^{1}\left(F_{n, 2}, \partial F_{n, 2} ; \mathbf{R}\right)$ are equipped with the (skew symmetric) intersection form which is naturally preserved by the action of $\tilde{h}_{\beta}^{\star}$. This intersection form is non degenerate on $H^{1}\left(F_{n, 2}, \partial F_{n, 2} ; \mathbf{R}\right)$ when $n$ is odd, so that one can identify $\mathbf{R}^{\infty}$ with the symplectic sum of an infinite number of copies of the canonical symplectic $\mathbf{R}^{2}$ in such a way that $H^{1}\left(F_{n, 2}, \partial F_{n, 2} ; \mathbf{R}\right)$ coincides with the sum of the first $(n-1) / 2$ copies when $n$ is odd.

It follows from Proposition 2.1 that when $n$ is odd, there is a symplectic structure on $\mathbf{R}^{n-1}$ which is invariant under the image of $\mathbf{B}_{n}$ by $\mathcal{B}_{-1}$. In other words, the representation $\mathcal{B}_{-1}$ has an image contained in a conjugate of the symplectic group $\mathbf{S p}(n-1, \mathbf{R})$. In the same way, up to conjugacy, one can assume that $\mathcal{B}_{-1}$ maps $\mathbf{B}_{\infty}$ into $\mathbf{S p}(\infty, \mathbf{R})$.

Consider now the case of a general root of unity $\omega$ of order $k \geq 3$. Again, the intersection form gives a symplectic structure on $H_{c}^{1}\left(F_{\infty, k} ; \mathbf{R}\right)$. Note that when $\operatorname{gcd}(k, n)=1$ one can consider $H^{1}\left(F_{n, k}, \partial F_{n, k} ; \mathbf{R}\right)$ as a symplectic subspace of $H_{c}^{1}\left(F_{\infty, k} ; \mathbf{R}\right)$ which is preserved by the action of $\mathbf{B}_{n}$. The orthogonal complement of this subspace is of course the cohomology with compact support of the complement $F_{\infty, k} \backslash F_{n, k}$ on which $\mathbf{B}_{n}$ acts trivially. In this way, 
we get a linear representation of $\mathbf{B}_{\infty}$ in the ascending union of the symplectic groups of $H^{1}\left(F_{n, k}, \partial F_{n, k} ; \mathbf{R}\right)$ for $\operatorname{gcd}(n, k)=1$. Observe that the eigenspace decomposition of $H^{1}\left(F_{n, k}, \partial F_{n, k} ; \mathbf{R}\right)$ is orthogonal with respect to the symplectic intersection form, so that we get in this way linear representations of $\mathbf{B}_{n}$ in the group of symplectic automorphisms of $H^{1}\left(F_{n, k}, \partial F_{n, k} ; \mathbf{R}\right)_{\omega}$.

It follows from Proposition 2.2 that when $\operatorname{gcd}(k, n)=1$, one can equip $\mathbf{R}^{2(n-1)}$ with a symplectic structure which is invariant by $\mathcal{B}_{\omega}$. Again, this means that, up to conjugacy, we can assume that $\mathcal{B}_{\omega}$ maps $\mathbf{B}_{\infty}$ in $\operatorname{Sp}(\infty, \mathbf{R})$.

To be complete, one should write the formula giving the symplectic form on $\mathbf{R}^{2 n-2}$ (or $\mathbf{R}^{n-1}$ when $\omega=-1$ ) coming from the intersection form via the isomorphisms given by Propositions 2.1 and 2.2. Since everything is very explicit, it is just the matter of a simple computation. Note that the intersection number of $t^{j} \cdot v_{i}^{k}$ and $t^{j} \cdot v_{i+1}^{k}$ (for $1 \leq i \leq n-2$ and $1 \leq j \leq k-1)$ is equal to +1 . The intersection number of $t^{j} \cdot v_{i}^{k}$ and $t^{j+1} \cdot v_{i}^{k}$ is equal to +1 ( $j$ defined modulo $k$ ). Finally, the intersection number of $t^{j} \cdot v_{i}^{k}$ and $t^{j+1} \cdot v_{i+1}^{k}$ is equal to -1 ( $j$ defined modulo $k$ ). All other intersection numbers are trivial.

From this, one gets the intersection form in the basis given by the $w_{i}^{k}$ 's. We shall skip this boring computation (or leave it to the reader!). Let us just mention that the result is coherent (as it should!) with the result of Squier [13] (see also [6]) that we now summarize. This author adds a formal variable $s$ with $s^{2}=t$ so that the $\operatorname{ring} \mathbf{Z}\left[t, t^{-1}\right]$ can be considered as a subring of $\mathbf{Z}\left[s, s^{-1}\right]$ and considers the involution of this ring sending $s$ to $s^{-1}$. He considers the transposed Burau matrices acting on $\mathbf{Z}\left[s, s^{-1}\right]^{n-1}$ and shows that they preserve the hermitian form $H_{s}$ with values in $\mathbf{Z}\left[s, s^{-1}\right]$, given by the matrix

$$
\left(\begin{array}{cccc}
s+s^{-1} & -s^{-1} & 0 & \vdots \\
-s & s+s^{-1} & -s^{-1} & \vdots \\
0 & -s & s+s^{-1} & \vdots \\
\cdots & \ldots & \cdots & .
\end{array}\right) .
$$

The determinant of $H_{s}$ is equal to $\left(s^{n}-s^{-n}\right) /\left(s-s^{-1}\right)$. When one specializes $t$ to be a complex number of modulus 1 and one chooses $s$ as one of the two square roots of $t$, the corresponding hermitian form reduces to a usual hermitian form which is invariant under the Burau matrices evaluated at $t$. Note that when $s$ and $t$ are specialized to complex numbers of modulus $1, H_{s}$ is singular if and only if $s$ is a $2 n$-th root of unity different from \pm 1 . If $\cos \frac{(l+1) \pi}{n}<\Re s<\cos \frac{l \pi}{n}$ for $0 \leq l \leq n-1$, the hermitian form $H_{s}$ has signature $n-1-2 l$.

We have two natural skew-symmetric forms which are invariant by $\mathcal{B}_{\omega}$ : 
the one coming from the intersection form in homology, and the imaginary part of $H_{s}$. The boring computation that we skipped shows that these forms are proportional and that the corresponding factor is $2 i k(s-\bar{s})$ so that if one chooses $s$ with a negative imaginary part, these two forms are related by a positive constant. If $\operatorname{gcd}(n, k)=1$, the surface $F_{n, k}$ has only one boundary component and its intersection form is non degenerate, i.e. is a symplectic form; this fits with the fact that in this situation $\omega$ is not an $n$-th root of unity and $H_{s}$ is non degenerate.

When the Burau representation is thought as a representation in the symplectic group, we call it the Burau-Squier representation.

\section{A visual computation}

\subsection{Signature of 4-manifolds}

Recall that the signature $\sigma(M)$ of an oriented compact 4-manifold is the signature of the intersection form on the second homology group $H_{2}(M ; \mathbf{R})$. Note that this intersection form can be degenerate if the boundary of $M$ is not empty: a class in $H_{2}(\partial M ; \mathbf{R})$ maps in $H_{2}(M ; \mathbf{R})$ to an element of the kernel of this form.

The signature of an oriented link can be seen as the signature of a 4-manifold as follows. Consider an oriented link $\lambda \subset \mathbf{S}^{3}$ and a Seifert surface $S_{\lambda}$ embedded in $\mathbf{S}^{3}$ which can be considered as the boundary of the 4-dimensional ball $\mathbf{D}^{4}$. Let us push the Seifert surface in $\mathbf{D}^{4}$ keeping its boundary fixed on $\mathbf{S}^{3}$ to get a surface $S_{\lambda}^{\prime}$ which intersects the boundary $\mathbf{S}^{3}$ only along its boundary. Consider the 2 -fold cover $V_{2}^{4}$ of $\mathbf{D}^{4}$ branched along $S_{\lambda}^{\prime}$. It is a 4-dimensional manifold whose boundary $\partial V_{2}^{4}$ is a 2 -fold cover over $\mathbf{S}^{3}$ branched along $\lambda$. It turns out (see for instance [7]) that:

$$
\operatorname{sign}(\lambda)=\sigma\left(V_{2}^{4}\right)
$$

In the general case of a root of unity $\omega$ which is different from -1 , we shall see in 3.4 that there is a similar interpretation of the $\omega$-signature of an oriented link in terms of signatures of 4-manifolds.

Remark: Ramified $\mathbf{Z} / k \mathbf{Z}$-cyclic coverings are omnipresent in this paper. Recall that such a covering is completely determined by a ramification locus (which is a codimension 2 submanifold) and a homomorphism from the fundamental group of its complement to $\mathbf{Z} / k \mathbf{Z}$. In the previous cases that we considered, the homomorphism was implicit. In the case of the ramified 2-fold cover of the disc, it was the unique homomorphism which is equal to 1 on small simple positive loops going once around the $x_{i}^{0}$ 's. In case of the 2 -fold cover of $\mathbf{S}^{3}$ ramified on an oriented link, it was the reduction modulo 2 of the linking number. In the case of the cover of $\mathbf{D}^{4}$ 
branched along $S_{\lambda}^{\prime}$, it was also given by a linking number: a curve in the complement of $S_{\lambda}^{\prime}$ bounds a disc in $\mathbf{D}^{4}$ and one considers the reduction modulo 2 of the intersection number of this disc with $S_{\lambda}^{\prime}$.

An important tool in our proof will be Novikov additivity theorem that we recall briefly now (see for instance [7] for a proof):

Theorem 3.1 (Novikov). Let $M$ be an oriented 4-manifold obtained by gluing two 4-manifolds $M_{1}$ and $M_{2}$ along some components of their boundaries. Then the signature is additive:

$$
\sigma(M)=\sigma\left(M_{1}\right)+\sigma\left(M_{2}\right) .
$$

(The orientations on $M_{1}$ and $M_{2}$ are of course induced by the orientation on $M$.)

In the proof of Theorem A, we shall need a more general situation where one glues manifolds with corners. Consider two oriented 4manifolds $M_{1}$ and $M_{2}$ with boundary. In the boundaries $\partial M_{1}, \partial M_{2}$, choose submanifolds, $V_{1}$ and $V_{2}$, of dimension 3 , possibly with boundary, and assume that we are given some homeomorphism $h$ from $V_{1}$ to $V_{2}$ reversing the orientations. Then one can glue $M_{1}$ and $M_{2}$ along $V_{1}$ and $V_{2}$ using $h$. This produces a new oriented 4-manifold $M$ with boundary.

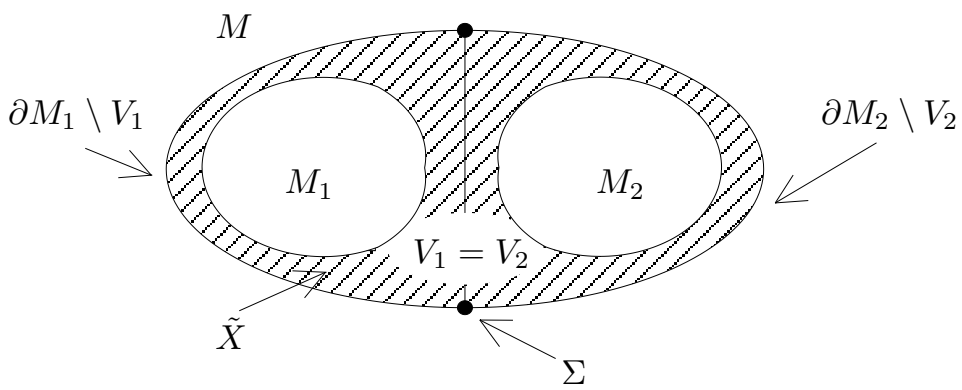

Figure 8: Gluing manifolds with corners

The Novikov additivity theorem does not apply in this situation: however one can write

$$
\sigma(M)=\sigma\left(M_{1}\right)+\sigma\left(M_{2}\right)+\delta
$$

where the "defect" $\delta$ is not difficult to analyze. Denote by $X$ the union of the boundaries of $M_{1}$ and $M_{2}$ in $M$. It consists of three 3-manifolds with boundaries having a common boundary: the closures of $\partial M_{1} \backslash V_{1}$ and $\partial M_{2} \backslash V_{2}$ (contained in the boundary of $M$ ) and $V_{1}$ (which is identified 
with $V_{2}$ in $\left.M\right)$. One can describe $M$ as the union of three 4-manifolds with boundary: $M_{1}, M_{2}$, and $\tilde{X}$, where $\tilde{X}$ denotes a regular neighborhood of $X$ in $M$. If follows from Novikov additivity theorem that $\delta$ is the signature of the oriented 4-manifold $\tilde{X}$ (see Figure 8). This means in particular that the defect only depends on the topology of $X$, i.e. on the boundaries of $M_{1}$ and $M_{2}$ and the gluing map. Let us make this defect more explicit.

Recall the notion of ternary Maslov index. Consider a real vector space $E$ equipped with a symplectic form $\Omega$, and three lagrangian subspaces $L_{1}, L_{2}, L_{3}$ (i.e. maximal isotropic subspaces). In the direct sum $L_{1} \oplus L_{2} \bigoplus L_{3}$ consider the subspace of triples $\left(x_{1}, x_{2}, x_{3}\right)$ whose sum is zero in $E$. On this subspace, one defines a symmetric bilinear form $f$ by

$$
\begin{aligned}
f\left(\left(x_{1}, x_{2}, x_{3}\right),\left(y_{1}, y_{2}, y_{3}\right)\right) & =\Omega\left(x_{1}, y_{3}\right)=\Omega\left(x_{2}, y_{1}\right)=\Omega\left(x_{3}, y_{2}\right) \\
& =\Omega\left(y_{1}, x_{3}\right)=\Omega\left(y_{2}, x_{1}\right)=\Omega\left(y_{3}, x_{2}\right) .
\end{aligned}
$$

The Maslov index $\tau\left(L_{1}, L_{2}, L_{3}\right)$ is by definition the signature of $f$ (see for instance [16] for a background). Note that this index is bounded by $3 \operatorname{dim} L=\frac{3}{2} \operatorname{dim} E$ (the better bound $\frac{1}{2} \operatorname{dim} E$ is possible but this will be enough for our needs).

The index $\tau\left(L_{1}, L_{2}, L_{3}\right)$ is a skew symmetric function of its three arguments and is a cocycle in the sense that for any four lagrangian subspaces, one has

$$
\tau\left(L_{1}, L_{2}, L_{3}\right)-\tau\left(L_{1}, L_{2}, L_{4}\right)+\tau\left(L_{1}, L_{3}, L_{4}\right)-\tau\left(L_{2}, L_{3}, L_{4}\right)=0 .
$$

In our situation, $X$ consists of three oriented 3-manifolds with a common boundary surface $\Sigma$. The first homology group $H_{1}(\Sigma, \mathbf{R})$ is a symplectic vector space (using intersection) as soon as one chooses an orientation on $\Sigma$ : let us choose the orientation that comes from the orientation of $\partial M_{1} \backslash V_{1}$ (which in turn comes from the orientation of $M_{1}$ ). In the symplectic vector space $H_{1}(\Sigma, \mathbf{R})$, one has three natural lagrangian subspaces $L_{1}, L_{2}, L_{3}$, corresponding to those homology classes in $\Sigma$ which are trivial when one maps them in the homology of $\partial M_{1} \backslash V_{1}, \partial M_{2} \backslash V_{2}$ and $V_{1}\left(\simeq V_{2}\right)$ respectively (these subspaces are lagrangian as follows from Poincaré duality). In [17], Wall proves that the defect $\delta$ is equal to the ternary Maslov index of these three lagrangian subspaces. This follows from a simple application of Mayer-Vietoris exact sequence which enables an identification of the intersection form in $H_{2}(\tilde{X}, \mathbf{R}$ ) (modulo subspaces contained in the kernel) to the quadratic form $f$ associated to $L_{1}, L_{2}, L_{3}$. We shall call this result the Novikov-Wall additivity theorem.

\subsection{The Meyer cocycle}

The Meyer cocycle evaluated on a pair of elements in $\mathbf{S p}(2 g, \mathbf{R})$ is also related to the signature of 4 -manifolds as follows. Let $P$ be the oriented 
pair of pants (the oriented 2-sphere minus three discs) and $W^{4}$ a 4-manifold which is an oriented fibration over $P$ whose fiber is a closed oriented surface $F$ with genus $g$ (the orientation on $M$ is induced by the orientations on $P$ and $F$ ).

It turns out that the signature of the oriented manifold $W^{4}$ only depends on the monodromy maps on the first homology group $H_{1}(F ; \mathbf{R})$ of the surface $F$.

The intersection form on $H_{1}(F ; \mathbf{R})$ is a symplectic form which is invariant under the action induced by homeomorphisms of $F$. Thus we can identify $H_{1}(F ; \mathbf{R})$ (equipped with the intersection form) to $\mathbf{R}^{2 g}$ (equipped with the standard symplectic form). This allows us to associate with each loop on the pair of pants $P$ (based at some base point in $P$ ) an element in $\mathbf{S p}(2 g, \mathbf{R})$.

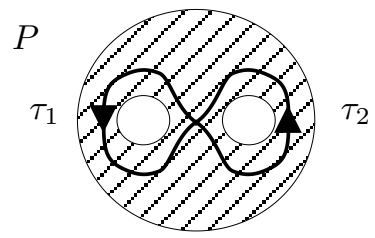

Figure 9: Pair of pants

The homology of $W^{4}$ can be computed from the associated spectral sequence. Consider the $E_{1,1}^{2}$ term of this spectral sequence, i.e. the first homology group $H_{1}\left(P ; H_{1}(F ; \mathbf{R})\right)$. The skew symmetric intersection on this homology

$$
H_{1}\left(P ; H_{1}(F ; \mathbf{R})\right) \otimes H_{1}\left(P ; H_{1}(F ; \mathbf{R})\right) \rightarrow H_{1}(F ; \mathbf{R}) \otimes H_{1}(F ; \mathbf{R})
$$

followed by the skew-symmetric intersection form on the coefficient module

$$
H_{1}(F ; \mathbf{R}) \otimes H_{1}(F ; \mathbf{R}) \rightarrow \mathbf{R}
$$

produces a real quadratic form $q$ on $H_{1}\left(P ; H_{1}(F ; \mathbf{R})\right)$. Meyer showed in [9] that the signature of $W^{4}$ is equal to the signature of this quadratic form $q$, which amounts to showing that the other terms in the spectral sequence do not contribute to the signature of $W^{4}$. In turn, the signature of $q$ can be easily computed from $\gamma_{1}$ and $\gamma_{2}$. Meyer's Theorem is the following:

Theorem 3.2 (Meyer). Let $\gamma_{1}$ and $\gamma_{2}$ be the two elements in $\mathbf{S p}(2 g, \mathbf{R})$ respectively induced by the two generators $\tau_{1}$ and $\tau_{2}$ of the fundamental group $\pi_{1}(P)$ (see Figure 9). The signature $\sigma\left(W^{4}\right)$ of the oriented manifold $W^{4}$ is given by the evaluation of the Meyer cocycle on the pair $\left(\gamma_{1}, \gamma_{2}\right)$ :

$$
\sigma\left(W^{4}\right)=\operatorname{Meyer}\left(\gamma_{1}, \gamma_{2}\right) \text {. }
$$


This fact is all we need for the proof of Theorem A and the reader might skip the rest of this subsection. However, we believe that it is useful to give a more conceptual definition of Meyer cocycle and its relationship with the signatures of fibrations over a pair of pants.

We first show that Meyer Theorem 3.2 follows from Novikov-Wall Theorem. If one splits the pair of pants in two halves as in Figure 10, the manifold $W$ appears as the union of two 4-manifolds glued along part of their boundaries, as discussed above. Each one of the two pieces has a signature equal to 0 since they are both homeomorphic to the product of a 3-manifold by an interval.

One is therefore led to evaluate the defect $\delta$ in this situation. In this case, the surface $\Sigma$ consists of two copies of $F$ and the three corresponding lagrangian subspaces of $H_{1}(F ; \mathbf{R}) \oplus H_{1}(F ; \mathbf{R})$ are the graphs of the identity, and of the actions of the two given diffeomorphisms in homology. The definition of Maslov ternary index $\delta$ reduces precisely to the definition of the Meyer cocycle that we gave in the introduction and we get indeed Meyer theorem from Novikov-Wall theorem.

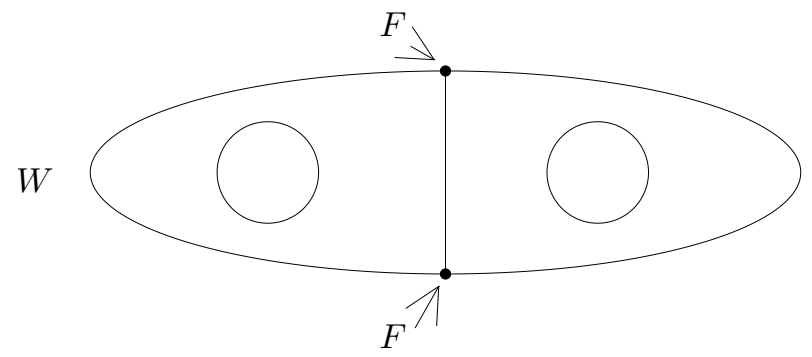

Figure 10: Splitting a pair of pants

We can at last give the final definition of Meyer cocycle.

Given a symplectic automorphism $\gamma$ of some symplectic vector space $(E, \Omega)$, its graph $\operatorname{Graph}(\gamma)=\{(x, \gamma(x))\} \subset E \times E$ is a lagrangian subspace of $E \times E$ equipped with the symplectic structure $\Omega \oplus-\Omega$. Hence, if we are given three symplectic automorphisms $\gamma_{1}, \gamma_{2}, \gamma_{3}$, we can consider the Maslov ternary index meyer $\left(\gamma_{1}, \gamma_{2}, \gamma_{3}\right)$ of $\left(\operatorname{Graph}\left(\gamma_{1}\right), \operatorname{Graph}\left(\gamma_{2}\right), \operatorname{Graph}\left(\gamma_{3}\right)\right)$. This defines a 2-cocycle in the homogeneous bar resolution since obviously meyer $\left(\gamma_{1}, \gamma_{2}, \gamma_{3}\right)=$ meyer $\left(\gamma \gamma_{1} \gamma^{\prime}, \gamma \gamma_{2} \gamma^{\prime}, \gamma \gamma_{3} \gamma^{\prime}\right)$ for every $\gamma, \gamma^{\prime}$ in the symplectic group of $(E, \Omega)$. The associated inhomogeneous cocycle is $\operatorname{Meyer}\left(\gamma_{1}, \gamma_{2}\right)=$ meyer $\left(i d, \gamma_{1}, \gamma_{1} \gamma_{2}\right)=\operatorname{meyer}\left(\gamma_{1}^{-1}, i d, \gamma_{2}\right)$. Going back to the definition of the Maslov ternary index, one sees that in order to compute $\operatorname{Meyer}\left(\gamma_{1}, \gamma_{2}\right)$, one should consider the space of triples $\left(v_{1}, \gamma_{1}^{-1}\left(v_{1}\right)\right),(v, v),\left(v_{2}, \gamma_{2}\left(v_{2}\right)\right)$ with sum 0 , i.e.

$$
v=-v_{1}-v_{2}
$$




$$
\left(\gamma_{1}^{-1}\left(v_{1}\right)-v_{1}\right)+\left(\gamma_{2}\left(v_{2}\right)-v_{2}\right)=0
$$

and one should compute one of the six possible (equal) symplectic products, one of them being

$(\Omega \oplus-\Omega)\left((v, v),\left(v_{1}, \gamma_{1}^{-1}\left(v_{1}\right)\right)=\Omega\left(v, v_{1}-\gamma_{1}^{-1}\left(v_{1}\right)\right)=\Omega\left(v_{1}+v_{2}, \gamma_{1}^{-1}\left(v_{1}\right)-v_{1}\right)\right.$.

One recovers the computational definition from the introduction.

Using the definition of Meyer cocycle with the ternary index, one immediately gets the symmetries:

$$
\begin{aligned}
\operatorname{Meyer}\left(\gamma_{1}, \gamma_{2}\right) & =\operatorname{Meyer}\left(\gamma_{2}, \gamma_{1}\right)=-\operatorname{Meyer}\left(\gamma_{1}^{-1}, \gamma_{2}^{-1}\right) \\
& =-\operatorname{Meyer}\left({ }^{t} \gamma_{1},{ }^{t} \gamma_{2}\right)=\operatorname{Meyer}\left(\gamma_{1},\left(\gamma_{1} \gamma_{2}\right)^{-1}\right)
\end{aligned}
$$

\subsection{Proof of Theorem $\mathrm{A}$ in the case $\omega=-1$}

We now begin the proof of the main Theorem A in the special case $\omega=-1$. The general case will be proved in the next subsection.

Before embarking in the proof, let us introduce a convenient notation. If $A$ and $B$ are numbers depending on some braids in $\mathbf{B}_{\infty}$, we shall write $A \cong B$ when $|A-B|$ is bounded by some universal constant (independent in particular of the braids).

Instead of proving Theorem A, we shall prove a loose version, i.e. that for any pair of braids $\alpha, \beta$, we have

$$
\operatorname{sign}(\widehat{\alpha \cdot \beta})-\operatorname{sign}(\hat{\alpha})-\operatorname{sign}(\hat{\beta}) \cong-\operatorname{Meyer}\left(\mathcal{B}_{-1}(\alpha), \mathcal{B}_{-1}(\beta)\right) .
$$

Let us first explain how this loose version almost immediately implies the sharp version where one replaces $\cong$ by $=$ in the previous relation.

Indeed, for every braid $\alpha$ in $\mathbf{B}_{n}$ and any integer $l \geq 1$, let us denote by $\alpha_{l}$ the element of $\mathbf{B}_{l n}$ consisting of a "juxtaposition" of $l$ copies of $\alpha$ : see Figure 11. Note that

$$
\operatorname{sign}\left(\widehat{\alpha_{l}}\right)=\operatorname{l.sign}(\widehat{\alpha})
$$

since a Seifert surface for $\widehat{\alpha_{l}}$ can be chosen as $l$ translated copies for a Seifert surface of $\hat{\alpha}$ and the corresponding Seifert matrix consists of $l$ diagonal blocks equal to the Seifert matrix associated to $\widehat{\alpha}$. 


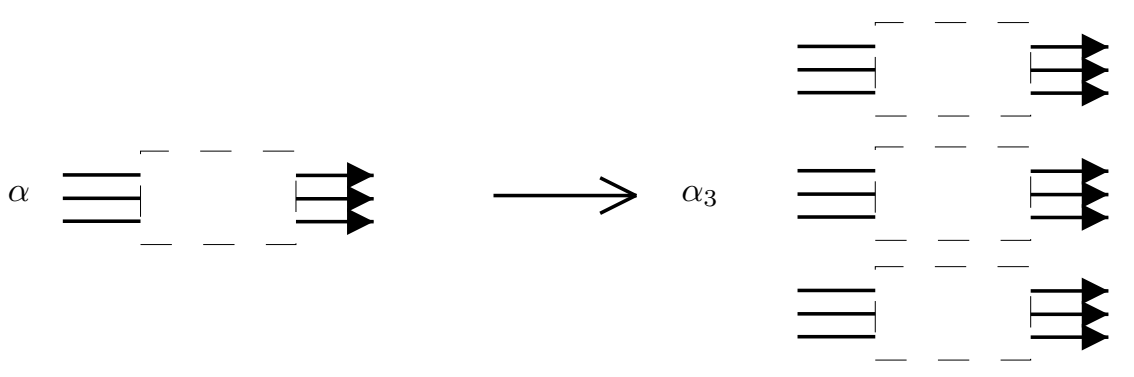

Figure 11: Juxtaposition of a braid

In the same way, $\mathcal{B}_{-1}\left(\alpha_{l}\right)$ can also be written as a block diagonal matrix, each of the $l$ blocks being a copy of $\mathcal{B}_{-1}(\alpha)$ (as can be seen directly in the Burau representation or in the action on the cohomology of $\left.F_{\infty, 2}\right)$. It follows that

$$
\operatorname{Meyer}\left(\mathcal{B}_{-1}\left(\alpha_{l}\right), \mathcal{B}_{-1}\left(\beta_{l}\right)\right)=\text { l.Meyer }\left(\mathcal{B}_{-1}(\alpha), \mathcal{B}_{-1}(\beta)\right) .
$$

If the quantity

$$
\operatorname{sign}(\widehat{\alpha \cdot \beta})-\operatorname{sign}(\hat{\alpha})-\operatorname{sign}(\hat{\beta})+\operatorname{Meyer}\left(\mathcal{B}_{-1}(\alpha), \mathcal{B}_{-1}(\beta)\right)
$$

were non zero, it would follow that

$$
\operatorname{sign}\left(\widehat{\alpha_{l} \cdot \beta_{l}}\right)-\operatorname{sign}\left(\widehat{\alpha_{l}}\right)-\operatorname{sign}\left(\widehat{\beta}_{l}\right)+\operatorname{Meyer}\left(\mathcal{B}_{-1}\left(\alpha_{l}\right), \mathcal{B}_{-1}\left(\beta_{l}\right)\right)
$$

is unbounded as $l$ tends to infinity. This would contradict the loose version of the theorem.

We now begin the proof of the "loose theorem". Let $\alpha$ and $\beta$ be two braids in $\mathbf{B}_{n}$. One can always assume that $n$ is odd so that $H^{1}\left(F_{n, 2}, \partial F_{n, 2} ; \mathbf{R}\right)$ is a symplectic subspace of $H_{c}^{1}\left(F_{\infty, 2} ; \mathbf{R}\right)$ and $\operatorname{Meyer}\left(\mathcal{B}_{-1}(\alpha), \mathcal{B}_{-1}(\beta)\right)$ can be computed using the representation of $\mathbf{B}_{n}$ on $H^{1}\left(F_{n, 2}, \partial F_{n, 2} ; \mathbf{R}\right)$. From now on, we denote by $\mathbf{D}^{2}$ a disc in the plane whose radius is obvious from the context or is not relevant.

Let $S_{2}$ be the oriented closed surface with genus 2 and consider the decomposition of $S_{2}$ in five pieces: two oriented pairs of pants $P$ and three oriented cylinders $C=\mathbf{S}^{1} \times[0,1]$, as shown in Figure 12 .

We shall construct a 4 -manifold with boundary, $M_{2}^{4}(\alpha, \beta)$, which is a 2fold cover over $S_{2} \times \mathbf{D}^{2}$ branched along some surface $\mathcal{S}(\alpha, \beta)$. This will be obtained by gluing together five manifolds with boundaries. The first two pieces, denoted $P_{2}^{4}(\alpha, \beta)$ and $P_{2}^{4}\left(e_{n}, e_{n}\right)$, will be 2 -fold branched covers over $P \times \mathbf{D}^{2}$. The three remaining pieces, denoted $C_{2}^{4}(\alpha), C_{2}^{4}(\beta)$ and $C_{2}^{4}(\alpha, \beta)$, will be 2 -fold branched covers over $C \times \mathbf{D}^{2}$. We now describe in detail the construction of each one of these pieces. 


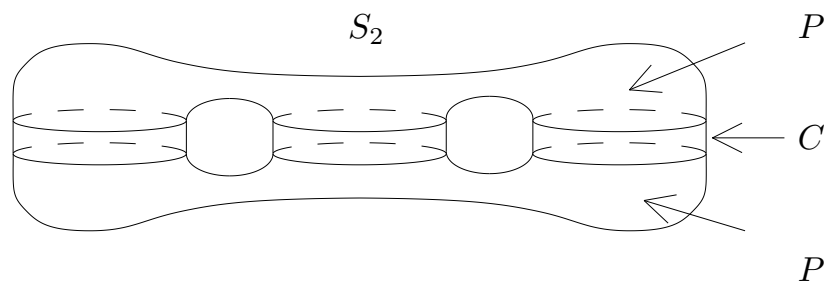

Figure 12: Surface of genus 2

- Construction of the manifolds $P_{2}^{4}(\alpha, \beta)$ and $P_{2}^{4}\left(e_{n}, e_{n}\right)$

Let $\tilde{h}_{\alpha}$ and $\tilde{h}_{\beta}$ be two homeomorphisms of $F_{n, 2}$ respectively associated to the two braids $\alpha$ and $\beta$ in $\mathbf{B}_{n}$. We consider an oriented 4-manifold $P_{2}^{4}(\alpha, \beta)$ which is a fibration over $P$ with fiber $F_{n, 2}$, such that the monodromy maps associated with the loops $\tau_{1}$ and $\tau_{2}$ in $\pi_{1}(P)$ are respectively $\tilde{h}_{\alpha}$ and $\tilde{h}_{\beta}$. The manifold $P_{2}^{4}(\alpha, \beta)$ can be seen as follows:

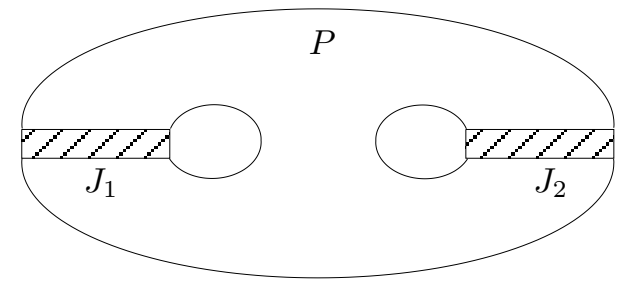

Figure 13: Cuts in the pair of pants

- On the oriented pair of pants $P$, we consider two cuts i.e. two intervals $I_{1}$ and $I_{2}$, and two neighborhoods $J_{1}=I_{1} \times[0,1]$ and $J_{2}=I_{2} \times[0,1]$ as in Figure 13.

- We consider two isotopies $\left\{h_{\alpha}^{t}\right\}_{t \in[0,1]}$ and $\left\{h_{\beta}^{t}\right\}_{t \in[0,1]}$ joining the identity map to $h_{\alpha}$ (resp. $h_{\beta}$ ) keeping the boundary of the 2 -disc pointwise fixed.

- We construct the surface $S(\alpha, \beta)$ in $P \times \mathbf{D}^{2}$ which coincides with - the surface $\left(P \backslash\left(J_{1} \cup J_{2}\right)\right) \times\left\{x_{1}^{0}, \ldots, x_{n}^{0}\right\}$ on $\left(P \backslash\left(J_{1} \cup J_{2}\right)\right) \times \mathbf{D}^{2}$, - the surface $\cup_{i=1}^{n}\left\{\left(x, t, h_{\alpha}^{t}\left(x_{i}^{0}\right)\right),(x, t) \in I_{1} \times[0,1]\right\}$ on $J_{1} \times \mathbf{D}^{2}$, - the surface $\cup_{i=1}^{n}\left\{\left(x, t, h_{\beta}^{t}\left(x_{i}^{0}\right)\right),(x, t) \in I_{2} \times[0,1]\right\}$ on $J_{2} \times \mathbf{D}^{2}$.

- In order to define $P_{2}^{4}(\alpha, \beta)$ as a 2 -fold cover over $P \times \mathbf{D}^{2}$ branched along the surface $S(\alpha, \beta)$ we still have to choose some homomorphism from the fundamental group of the complement $P \times \mathbf{D}^{2} \backslash S(\alpha, \beta)$ to $\mathbf{Z} / 2 \mathbf{Z}$. We choose the only homomorphism which is trivial when restricted to loops contained in $P \times\{x\}$ (with $x \in \partial \mathbf{D}^{2}$ ) and which is equal to 1 on small simple positive loops contained in $\{p\} \times\left(\mathbf{D}^{2} \backslash\left\{x_{1}^{0}, \ldots, x_{n}^{0}\right\}\right)$ going once around $x_{i}^{0}$ (for $p \in P \backslash\left(J_{1} \cup J_{2}\right)$ ). The existence and uniqueness of such a 
homomorphism is easy to check. The orientation of the manifold $P_{2}^{4}(\alpha, \beta)$ is induced by the orientation of $P \times \mathbf{D}^{2}$.

Notice that the boundary $\partial P_{2}^{4}(\alpha, \beta)$ consists of two parts (see Figure 14).

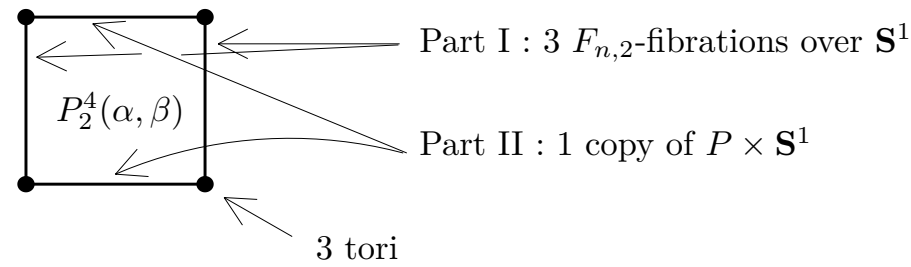

Figure 14: The manifold $P_{2}^{4}(\alpha, \beta)$

The first is the union of the total spaces of three fibrations over the circle with a fiber homeomorphic to $F_{n, 2}$, the monodromies being $\tilde{h}_{\alpha}, \tilde{h}_{\alpha}, \tilde{h}_{\alpha \cdot \beta}$ (where $\tilde{h}_{\alpha \cdot \beta}=\tilde{h}_{\beta} \circ \tilde{h}_{\alpha}$ ). The second part is one copy of $P \times \mathbf{S}^{1}$ (since $n$ is odd). These two parts intersect along the disjoint union $\Sigma$ of three tori $\mathbf{S}^{1} \times \mathbf{S}^{1}$. Notice also that the three fibrations that constitute the first part of the boundary of $P_{2}^{4}(\alpha, \beta)$ can be seen as 2-fold covers over the solid torus $T=\mathbf{S}^{1} \times \mathbf{D}^{2}$ branched along the links $\hat{\alpha}, \hat{\beta}$ and $\widehat{\alpha \cdot \beta}$ in $T$.

The manifold $P_{2}^{4}\left(e_{n}, e_{n}\right)$ is constructed in the same way by replacing the braids $\alpha$ and $\beta$ by the trivial braid $e_{n}$. More simply:

$$
P_{2}^{4}\left(e_{n}, e_{n}\right)=P \times F_{n, 2} .
$$

\section{Lemma 3.3.}

$$
\sigma\left(P_{2}^{4}(\alpha, \beta)\right) \cong-\operatorname{Meyer}\left(\mathcal{B}_{-1}(\alpha), \mathcal{B}_{-1}(\beta)\right)
$$

Proof. Gluing one copy of $P \times \mathbf{D}^{2}$ along the boundary of $P_{2}^{4}(\alpha, \beta)$ we get a manifold $\tilde{P}_{2}^{4}(\alpha, \beta)$ which is a fibration over $P$ with fiber a closed surface $\tilde{F}_{n, 2}$ (with the same first homology as $F_{n, 2}$ since $n$ is odd). The signature of this second manifold is exactly $-\operatorname{Meyer}\left(\mathcal{B}_{-1}(\alpha), \mathcal{B}_{-1}(\beta)\right)$. Indeed this signature is equal to the Meyer cocycle evaluated on the action in homology and the definition of $\mathcal{B}_{-1}$ uses cohomology : this amounts to a change of sign. Note that we use Meyer Theorem 3.2 and the topological interpretation of $\mathcal{B}_{-1}$ given in 2.1 .

We now use Novikov-Wall additivity theorem so that the signature of $P_{2}^{4}(\alpha, \beta)$ and $\operatorname{Meyer}(\mathcal{B}(\alpha), \mathcal{B}(\beta))$ differ by some defect $\delta$ which is uniformly bounded since we saw that the corresponding surface $\Sigma$ consists of three tori.

- The construction of the manifolds $C_{2}^{4}(\alpha), C_{2}^{4}(\beta)$ and $C_{2}^{4}(\alpha, \beta)$ 
Consider the solid torus $T=\mathbf{S}^{1} \times \mathbf{D}^{2}$ introduced above that we can think of as embedded in the 3 -sphere $\mathbf{S}^{3}$ in the standard way. Let $\alpha$ be a braid in $\mathbf{B}_{n}$ and $\hat{\alpha}$ the associated closed braid in $T$.

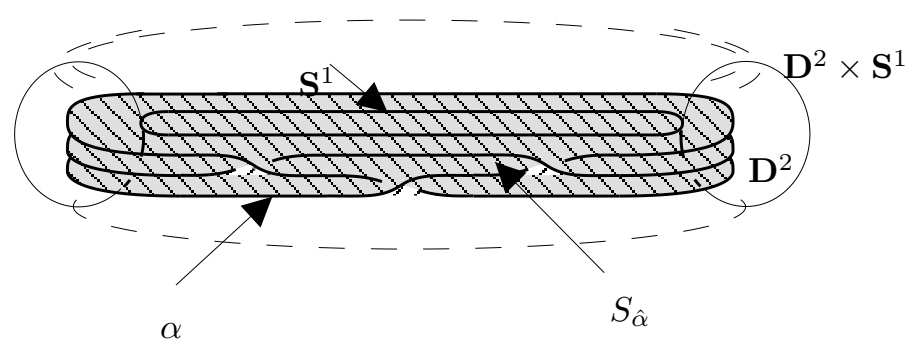

Figure 15: Seifert surface

For each word in the $\sigma_{i}^{ \pm 1}$ 's representing $\alpha$, one can associate a Seifert surface $S_{\hat{\alpha}}$ for the closed link which cuts the boundary $\partial T=\mathbf{S}^{1} \times \partial \mathbf{D}^{2}$ along $n$ parallel circles (see Figure 15). It consists of $n$ parallel discs connected by half twisted bands, each band corresponding to a letter in the word, and the sense of the twisting depending of the exponent of the letter.

Consider the unit ball $\mathbf{D}^{4}$ in $\mathbf{R}^{4}$, oriented in a compatible way with its boundary $\partial \mathbf{D}^{4}=\mathbf{S}^{3}$. From the spherical shell $\left\{x \in \mathbf{D}^{4} ; 1 / 2 \leq\|x\| \leq 1\right\}$, we extract the oriented 4-manifold with boundary

$$
\mathbf{N}^{4}=\left\{x \in \mathbf{D}^{4} ; 1 / 2 \leq\|x\| \leq 1, \text { and } \mathrm{x} /\|\mathrm{x}\| \in \mathrm{T}\right\}
$$

which is homeomorphic to $T \times[0,1]$. One can push the surface $S_{\hat{\alpha}}$ towards the interior of $\mathbf{D}^{4}$ so that it now satisfies

- the boundary $\partial S_{\hat{\alpha}}$ is in $T \subset \mathbf{S}^{3}=\partial \mathbf{B}^{4}$ and is equal to $\hat{\alpha}$,

$-S_{\hat{\alpha}}$ does not intersect the set of points $x$ in $\partial \mathbf{N}^{4}$ such that $x /\|x\|$ is in $\partial T ;$

- $S_{\hat{\alpha}}$ intersects the sphere $\left\{x \in \mathbf{D}^{4} ;\|x\|=1 / 2\right\}$ in a trivial link with $n$ components,

$-S_{\hat{\alpha}} \cap\left(\mathbf{D}^{4} \backslash \mathbf{N}^{4}\right)$ is a collection of $n$ disjoint discs contained in the ball $\left\{x \in \mathbf{D}^{4} ;\|x\| \leq 1 / 2\right\}$,

- The circle $\mathbf{S}^{1}$ can be equipped with an origin 0 such that $S_{\hat{\alpha}} \cap\{0\} \times$ $[0,1] \times \mathbf{D}^{2}=\{0\} \times[0,1] \times\left\{x_{1}^{0}, \ldots, x_{n}^{0}\right\}$.

The Seifert surface $S_{\hat{\alpha}}$ being in such a position, we denote by $S^{c}(\alpha)$ the intersection surface $S_{\hat{\alpha}} \cap \mathbf{N}^{4}$. 


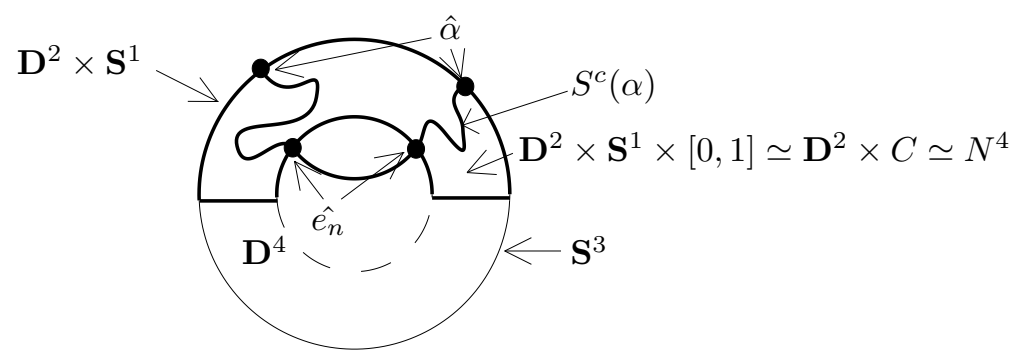

Figure 16: Pushing the Seifert surface in 4-ball

The 4-manifold $C_{2}^{4}(\alpha)$ is defined as a 2-fold cover over $C \times \mathbf{D}^{2} \simeq N^{4}$ branched along $S^{c}(\alpha)$. One should specify some homomorphism from the fundamental group of the complement of $S^{c}(\alpha)$ in $N^{4}$ to $\mathbf{Z} / 2 \mathbf{Z}$ : one simply takes the one induced by the linking number with $S_{\hat{\alpha}}$ in $\mathbf{D}^{4}$. Note that $C_{2}^{4}(\alpha)$ fits

- with the 2 -fold cyclic cover over the solid torus $\mathbf{S}^{1} \times\{0\} \times \mathbf{D}^{2}$ branched along the link $\hat{\alpha}$,

- with the 2 -fold cyclic cover over the solid torus $\mathbf{S}^{1} \times\{1\} \times \mathbf{D}^{2}$ branched along the trivial link $\hat{e}_{n}$.

These two last ramified covers are associated to the homomorphism in $\mathbf{Z} / 2 \mathbf{Z}$ given by the reduction modulo 2 of the linking number, when one sees the solid torus as embedded in the 3 -sphere in the usual way. The orientation of the manifold $C_{2}^{4}(\alpha)$ is induced by the orientation of the manifold $C \times \mathbf{D}^{2}$.

Our notation suggests that $C_{2}^{4}(\alpha)$ only depends on $\alpha$; this is not the case since it depends on the choice of the Seifert surface which depends in turn on the choice of a word in the $\sigma_{i}^{ \pm 1}$ representing $\alpha$. However, for any braid $\alpha$, we choose such a word once for all in an arbitrary way, and we shall denote by $C_{2}^{4}(\alpha)$ the manifold obtained using this word.

Lemma 3.4.

$$
\sigma\left(C_{2}^{4}(\alpha)\right) \cong \operatorname{sign}(\hat{\alpha})
$$

Proof. Again, it follows from Novikov-Wall additivity theorem. Indeed let $V_{2}^{4}(\alpha)$ be the double cover of $\mathbf{D}^{4}$ branched along $S_{\hat{\alpha}} \subset \mathbf{D}^{4}$ with signature $\operatorname{sign}(\hat{\alpha})$. On gets $V_{2}^{4}(\alpha)$ from $C_{2}^{4}(\alpha)$ by gluing first one copy of $\mathbf{S}^{1} \times[0,1] \times \mathbf{D}^{2}$ on one part of the boundary and then $V_{2}^{4}\left(\hat{e}_{n}\right)$ along the new boundary (see Figure 16). As before, the defect in Novikov-Wall additivity is uniformly bounded.

Finally, we define $C_{2}^{4}(\alpha, \beta)$ as the manifold constructed exactly in the same way as $C_{2}^{4}(\alpha \cdot \beta)$, except that, as a word representing $\alpha \cdot \beta$ we use the concatenation of the chosen words representing $\alpha$ and $\beta$. Of course $\sigma\left(C_{2}^{4}(\alpha, \beta)\right) \cong \operatorname{sign}(\widehat{\alpha \cdot \beta})$. 


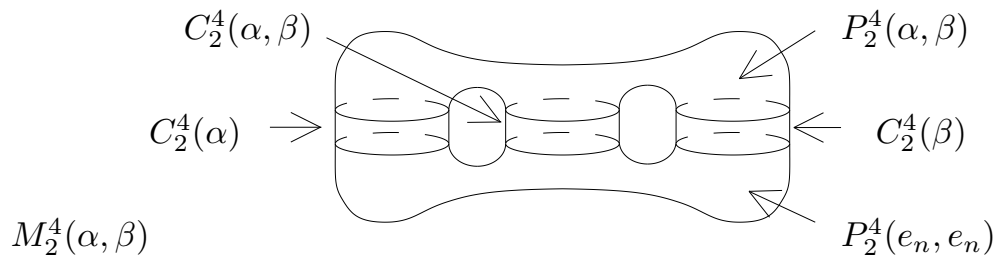

Figure 17: The manifold $M_{2}^{4}(\alpha, \beta)$

The manifold $M_{2}^{4}(\alpha, \beta)$ is obtained by gluing together the manifolds $C_{2}^{4}(\alpha), C_{2}^{4}(\beta)$ and $C_{2}^{4}(\alpha, \beta)$ with $P_{2}^{4}(\alpha, \beta)$ on one side and $P_{2}^{4}\left(e_{n}, e_{n}\right)$ on the other side. The manifold $M_{2}^{4}(\alpha, \beta)$ is a 2 -fold cover of the manifold $P \times \mathbf{D}^{2}$ branched over the surface $\mathcal{S}(\alpha, \beta)$ which is obtained by gluing $S^{c}(\alpha), S^{c}(\beta)$ and $S^{c}(\alpha \beta)$ with $S(\alpha, \beta)$ on one side and $S\left(e_{n}, e_{n}\right)$ on the other side (see Figure 17).

The orientation of the manifold $P \times \mathbf{D}^{2}$ induces an orientation of the manifold $P_{2}^{4}(\alpha, \beta)$ which induces in turn an orientation on the manifold $M_{2}^{4}(\alpha, \beta)$. This orientation also fits with the orientations of $C_{2}^{4}(\alpha, \beta)$ and $P_{2}^{4}\left(e_{n}, e_{n}\right)$, but does not fit with the orientations of $C_{2}^{4}(\alpha)$ and $C_{2}^{4}(\beta)$. From the Novikov-Wall additivity theorem and the observation that the defects $\delta$ associated to the gluing maps is uniformly bounded, we get:

\section{Lemma 3.5.}

$\sigma\left(M_{2}^{4}(\alpha, \beta)\right) \cong \operatorname{sign}(\widehat{\alpha \cdot \beta})-\operatorname{sign}(\hat{\alpha})-\operatorname{sign}(\hat{\beta})+\operatorname{Meyer}\left(\mathcal{B}_{1}(\alpha), \mathcal{B}_{1}(\beta)\right)$.

In order to prove Theorem A, we have to show:

\section{Lemma 3.6.}

$$
\sigma\left(M_{2}^{4}(\alpha, \beta)\right) \cong 0
$$

Let us revisit the construction of the manifold $C_{2}^{4}(\alpha, \beta)$. Since it uses a representation of $\alpha \cdot \beta$ as a concatenation of two words representing $\alpha$ and $\beta$, the Seifert surface used in the construction consists in gluing every disc associated with $\hat{\alpha}$ with a disc associated with $\hat{\beta}$ along an interval along their boundary (see Figure 18). 


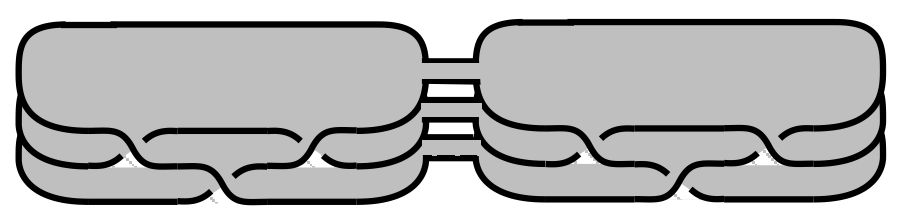

Figure 18: Seifert surface for a product of braids

It follows that one can choose on the circle $\mathbf{S}^{1}$ identified with $\mathbf{R} / \mathbf{Z}$ (already equipped with an origin 0 ), a second point, say $1 / 2$ such that $S^{c}(\alpha \cdot \beta) \cap\{1 / 2\} \times[0,1] \times \mathbf{D}^{2}=\{1 / 2\} \times[0,1] \times\left\{x_{1}^{0}, \ldots, x_{n}^{0}\right\}$. Thus, it is possible to cut the surface $S_{2}$ along a simple closed curve $c$ as described in Figure 19 such that the manifold $c \times \mathbf{D}^{2}$ intersects the surface $\mathcal{S}(\alpha, \beta)$ in $S_{2} \times \mathbf{D}^{2}$ along the $n$ parallel circles $c \times\left\{x_{1}^{0}, \ldots, x_{n}^{0}\right\}$.

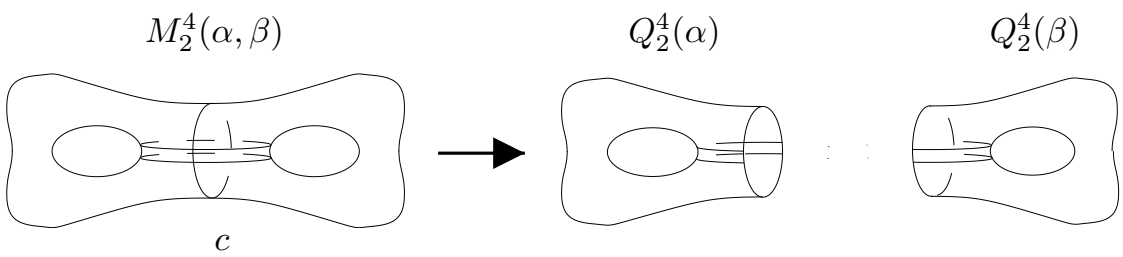

Figure 19: Splitting in two

It follows that the manifold $M_{2}^{4}(\alpha, \beta)$ can be cut along the 3 -manifold with boundary $c \times F_{n, 2}$ in two manifolds with boundary $Q_{2}^{4}(\alpha)$ and $Q_{2}^{4}(\beta)$ and thus:

$$
\sigma\left(M_{2}^{4}(\alpha, \beta)\right) \cong \sigma\left(Q_{2}^{4}(\alpha)\right)+\sigma\left(Q_{2}^{4}(\beta)\right)
$$

(note again that the defect is bounded). Observe that, as suggested by the notation, the manifold $Q_{2}^{4}(\alpha)$ only depends on the braid $\alpha$ (always keeping the same arbitrary choice of a word representing each braid).

If one chooses as $\beta$ the trivial braid $e_{n}$, this gives

$$
\sigma\left(M_{2}^{4}\right)\left(\alpha, e_{n}\right) \cong \sigma\left(Q_{2}^{4}(\alpha)\right)+\sigma\left(Q_{2}^{4}\left(e_{n}\right)\right) .
$$

By Lemma 3.5, the left hand side of this equality is equal to $\operatorname{Meyer}\left(\mathcal{B}_{1}(\alpha), i d\right)=0$ so that $\sigma\left(Q_{2}^{4}(\alpha)\right) \cong 0$. We finally conclude that $\sigma\left(M_{2}^{4}(\alpha, \beta)\right) \cong 0$. This finishes the proof of Lemma 3.6 and thus the proof of Theorem A.

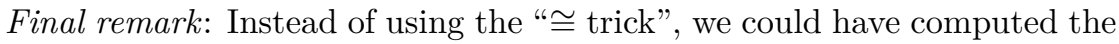
exact values of each defect $\delta$ for each of our gluing maps. This is indeed possible but rather cumbersome. 


\subsection{Equivariant signature of 4-manifolds: proof of Theorem $\mathrm{A}$ in the general case}

We first explain in concrete terms a special case of a general construction due to Atiyah and Singer [2].

Let $M^{4}$ be an oriented 4-manifold equipped with an (orientation preserving) action of $\mathbf{Z} / k \mathbf{Z}$ (which will be abbreviated by "an oriented $\mathbf{Z} / k \mathbf{Z}$-manifold"). As before, the real vector space $H_{2}\left(M^{4} ; \mathbf{R}\right)$ can be decomposed as a sum of subspaces $H_{2}\left(M^{4} ; \mathbf{R}\right)_{\omega}$ associated to the $k$-th roots of unity $\omega$ (subspaces associated to conjugate roots are equal). This decomposition is obviously orthogonal with respect to the intersection form. We denote by $\sigma_{\omega}$ the signature of the restriction of the intersection form to the subspace associated to $\omega$. For each $k$-th root of unity $\omega$, this defines a $\omega$-signature $\sigma_{\omega}\left(M^{4}\right)$ of the $\mathbf{Z} / k \mathbf{Z}$-manifold $M^{4}$.

In this context of $\mathbf{Z} / k \mathbf{Z}$-manifolds, the Novikov additivity theorem extends. If a 4-manifold $M$ can be written as a union of two 4-manifolds $M_{1}$ and $M_{2}$ glued along some boundary components and if there is an action of $\mathbf{Z} / k \mathbf{Z}$ which respects this decomposition, then for each $k$-th root of unity $\omega$, we have $\sigma_{\omega}(M)=\sigma_{\omega}\left(M_{1}\right)+\sigma_{\omega}\left(M_{2}\right)$. The proof is exactly the same as the proof of the classical case and one finds a slightly different (but equivalent) statement in [7, 17]. In the same way, the NovikovWall generalization also extends trivially (if one defines the defect as the Maslov index of the associated lagrangian subspaces on the corresponding $\omega$-subspace of $H_{1}(\Sigma, \mathbf{R})$, see [17]).

Consider an oriented link $\lambda \subset \mathbf{S}^{3}$ and a Seifert surface $S_{\lambda}$ embedded in $\mathbf{S}^{3}$. Let us push the Seifert surface in $\mathbf{D}^{4}$ keeping its boundary fixed on $\mathbf{S}^{3}$ to get a surface $S_{\lambda}^{\prime}$ which intersects the boundary $\mathbf{S}^{3}$ only along its boundary. Consider the $k$-fold cover $V_{k}^{4}$ of $\mathbf{D}^{4}$ branched along $S_{\lambda}^{\prime}$ (associated to the obvious homomorphism in $\mathbf{Z} / k \mathbf{Z}$ given by reduction modulo $k$ of the linking number). It is a 4 -dimensional manifold whose boundary $\partial V_{k}^{4}$ is a $k$-fold cover over $\mathbf{S}^{3}$ branched along $\lambda$. It turns out (see for instance [7]) that for every $k$-th root of unity $\omega$ :

$$
\operatorname{sign}_{\omega}(\lambda)=\sigma_{\omega}\left(V_{k}^{4}\right) .
$$

Similarly, there is an equivariant formulation for the signature of fibrations over the pair of pants. If a 4-manifold $W^{4}$ fibers over $P$ with a closed surface $F$ as a fiber and if one has an action of $\mathbf{Z} / k \mathbf{Z}$ on $W^{4}$ preserving each fiber, then for each $k$-th root of unity $\omega$, one can compute the $\omega$-signature of $W^{4}$ by considering first $H_{1}\left(P ; H_{1}(F)\right)$ as a $\mathbf{Z} / k \mathbf{Z}$-vector space and then by computing the signature of the restriction of the quadratic form to the corresponding $\omega$-subspace. The symplectic automorphisms of $H_{1}(F ; \mathbf{R})$ associated to the two loops $\tau_{1}$ and $\tau_{2}$ commute with the action of $\mathbf{Z} / k \mathbf{Z}$ and so define symplectic automorphisms $\left(\gamma_{1}\right)_{\omega}$ and $\left(\gamma_{2}\right)_{\omega}$ of $H_{1}(F ; \mathbf{R})_{\omega}$. As one can expect, there is an equivariant version 
of Meyer Theorem 3.2: the $\omega$-signature of the $\mathbf{Z} / k \mathbf{Z}$-manifold $W^{4}$ is equal to the Meyer cocycle evaluated on the two linear automorphisms $\left(\gamma_{1}\right)_{\omega}$ and $\left(\gamma_{2}\right)_{\omega}$. This follows easily from the equivariant version of NovikovWall Theorem (with the same proof as in the non equivariant case, given in 2.3).

Choose two braids $\alpha, \beta$ in $\mathbf{B}_{n}$. Assume, with no loss of generality, that $\operatorname{gcd}(n, k)=1$. This defines as before a fibration $P_{k}^{4}(\alpha, \beta)$ over the pair of pants $P$ with fiber the closed surface $\tilde{F}_{n, k}$ obtained from $F_{n, k}$ by gluing a disc to the boundary. There is a natural action of $\mathbf{Z} / k \mathbf{Z}$ on $P_{k}^{4}(\alpha, \beta)$. The $\omega$-signature of $P_{k}^{4}(\alpha, \beta)$ is equal to $-\operatorname{Meyer}\left(\mathcal{B}_{\omega}(\alpha), \mathcal{B}_{\omega}(\beta)\right)$.

The end of the proof of Theorem $\mathrm{A}$ is now clear. One just repeats the "cut and paste" arguments of Section 3, replacing all double covers by $k$-fold cyclic covers, considering all manifolds as $\mathbf{Z} / k \mathbf{Z}$-manifolds, and replacing usual signatures by the $\omega$-signatures. All arguments go through with no modification.

\section{Braids with three strands}

In this section, we analyze in detail the situation when $n=3$. We shall get formulas for the signature of 3-braids, extending some preliminary computations (by very different methods) from [10].

\subsection{Some computations}

One finds in [3] several interpretations of the Meyer cocycle in terms of cyclic orderings. As a warm up for our study of $\mathbf{B}_{3}$, we give some simple formulas for $\operatorname{Meyer}\left(\gamma_{1}, \gamma_{2}\right)$ where $\gamma_{1}$ and $\gamma_{2}$ are elements of $\mathbf{S p}(2, \mathbf{R})=$ $\mathbf{S L}(2, \mathbf{R})$.

Suppose first that $\gamma_{1}$ and $\gamma_{2}$ are non parabolic, i.e. that $\gamma_{1}-i d$ and $\gamma_{2}-i d$ are invertible (or equivalently that $\operatorname{trace}\left(\gamma_{1}\right) \neq 2$ and $\operatorname{trace}\left(\gamma_{2}\right) \neq 2$ ). Then, Meyer $\left(\gamma_{1}, \gamma_{2}\right)$ is the signature of the quadratic form $q_{\gamma_{1}, \gamma_{2}}$ on $\mathbf{R}^{2}$ defined by

$$
q_{\gamma_{1}, \gamma_{2}}(e)=\operatorname{det}\left(\left(\gamma_{1}^{-1}-i d\right)^{-1}(e)+\left(i d-\gamma_{2}\right)^{-1}(e), e\right) .
$$

A simple computation leads to the following more symmetric formula:

$$
q_{\gamma_{1}, \gamma_{2}}(e)=\operatorname{det}\left(\left(\frac{1}{2-\operatorname{trace}\left(\gamma_{1}\right)} \cdot \gamma_{1}+\frac{1}{2-\operatorname{trace}\left(\gamma_{2}\right)} \cdot \gamma_{2}\right)(e), e\right) .
$$

The discriminant of $q_{\gamma_{1}, \gamma_{2}}$ is equal to $4 \frac{\left(\operatorname{trace}\left(\gamma_{1} \gamma_{2}\right)-2\right)}{\left(\operatorname{trace}\left(\gamma_{1}\right)-2\right)\left(\operatorname{trace}\left(\gamma_{2}\right)-2\right)}$ as the reader will check readily.

For instance, if $\gamma_{2}=-i d$ and if $\gamma_{1}$ is hyperbolic (i.e. $\mid$ trace $\left(\gamma_{1}\right) \mid \neq 2$ ), one sees that the two eigenvectors of $\gamma_{1}$ are isotropic for $q_{\gamma_{1}, \gamma_{2}}$. Hence for 
all hyperbolic $\gamma$, we have

$$
\operatorname{Meyer}(\gamma,-i d)=0 .
$$

In the same way, if $\gamma$ is hyperbolic,

$$
\operatorname{Meyer}\left(\gamma^{p_{1}}, \gamma^{p_{2}}\right)=0
$$

since eigenvectors of $\gamma$ are isotropic for $q_{\gamma^{p_{1}}, \gamma^{p_{2}}}$.

Assume now that $\gamma_{1}$ is non trivial parabolic and that $\gamma_{2}$ is non parabolic. Up to conjugacy, we can assume that $\gamma_{1}=\left(\begin{array}{ll}1 & \epsilon \\ 0 & 1\end{array}\right)$ where $\epsilon= \pm 1$. The vector space $E_{\gamma_{1}, \gamma_{2}}=\operatorname{Im}\left(\gamma_{1}^{-1}-i d\right) \cap \operatorname{Im}\left(\gamma_{2}-i d\right)$ is spanned by $e_{1}=(1,0)$. The corresponding quadratic form $q_{\gamma_{1}, \gamma_{2}}$ is

$$
q_{\gamma_{1}, \gamma_{2}}\left(e_{1}\right)=\operatorname{det}\left(\left(0, \epsilon^{-1}\right)+\left(i d-\gamma_{2}\right)^{-1}(1,0),(1,0)\right)=\epsilon \frac{2-\operatorname{trace}\left(\gamma_{1} \gamma_{2}\right)}{2-\operatorname{trace}\left(\gamma_{2}\right)}
$$

as a simple calculation shows. Hence $\operatorname{Meyer}\left(\gamma_{1}, \gamma_{2}\right)$ is $0,+1,-1$ according to the sign of the latter expression.

Finally, if both $\gamma_{1}$ and $\gamma_{2}$ are parabolic, it is easy to see that $\operatorname{Meyer}\left(\gamma_{1}, \gamma_{2}\right)=0$ unless $\gamma_{1}$ and $\gamma_{2}$ have a common fixed vector. In the latter case, up to conjugacy, one has to compute $\operatorname{Meyer}\left(\left(\begin{array}{cc}1 & t_{1} \\ 0 & 1\end{array}\right),\left(\begin{array}{cc}1 & t_{2} \\ 0 & 1\end{array}\right)\right)$. The result is $0, \pm 1$ according to the sign of $\frac{1}{t_{1}}+\frac{1}{t_{2}}$.

\subsection{Signatures in $\mathrm{B}_{3}$}

Recall that the group $\mathbf{B}_{n}$ has an infinite center generated by the square of the element

$$
\Delta_{n}=\left(\sigma_{1} \cdot \sigma_{2} \cdots \sigma_{n-1}\right) \cdot\left(\sigma_{1} \cdots \sigma_{n-2}\right) \cdots\left(\sigma_{1} \cdot \sigma_{2}\right) \cdot \sigma_{1} .
$$

One can also write $\Delta_{n}^{2}$ as $\left(\sigma_{1} \cdots \sigma_{n-1}\right)^{n}$.

Let us first consider the case of the ordinary signature (i.e. $\omega=-1$ ). The double cover of a disc ramified in three points is a torus with one hole so that the closed surface $\tilde{F}_{3,2}$ is homeomorphic to a torus. Since the representation $\mathcal{B}_{-1}: \mathbf{B}_{3} \rightarrow \mathbf{S p}(2, \mathbf{R})=\mathbf{S L}(2, \mathbf{R})$ is trivial on $\Delta_{3}^{4}$, it induces a homomorphism from $\mathbf{B}_{3} / \mathbf{Z} . \Delta_{3}^{4}$ to $\mathbf{S L}(2, \mathbf{R})$. Of course, this is an isomorphism between $\mathbf{B}_{3} / \mathbf{Z} . \Delta_{3}^{4}$ and $\mathbf{S L}(2, \mathbf{Z})$, mapping $\sigma_{1}$ and $\sigma_{2}$ to the unipotent matrices $\left(\begin{array}{cc}1 & 0 \\ -1 & 1\end{array}\right)$ and $\left(\begin{array}{ll}1 & 1 \\ 0 & 1\end{array}\right)$ respectively.

Lemma 4.1. For any braid $\alpha$ in $\mathbf{B}_{n}$, one has $\operatorname{sign}\left(\alpha \cdot \Delta_{n}^{4}\right)=\operatorname{sign}(\alpha)-$ $n^{2}+1$. 
Proof. By Theorem A, the difference $\operatorname{sign}\left(\alpha \cdot \Delta_{n}^{4}\right)-\operatorname{sign}(\alpha)-\operatorname{sign}\left(\Delta_{n}^{4}\right)$ is equal to $-\operatorname{Meyer}\left(\mathcal{B}_{-1}(\alpha), \mathcal{B}_{-1}\left(\Delta_{n}^{4}\right)\right)=-\operatorname{Meyer}\left(\mathcal{B}_{-1}(\alpha), i d\right)$. The computational (as well as the topological) definition of the Meyer cocycle shows that $\operatorname{Meyer}\left(\mathcal{B}_{-1}(\alpha), i d\right)=0$. The lemma follows from the fact that according to [11], the signature of $\Delta_{n}^{4}$ is equal to $1-n^{2}$. This numerical value will also be deduced from a more general computation in the next subsection.

Let us denote by $l k_{n}: \mathbf{B}_{n} \rightarrow \mathbf{Z}$ the homomorphism sending each generator to 1 . It follows from the previous lemma that $s i g n+\frac{n+1}{2 n} l k_{n}$ descends to a map from $\mathbf{B}_{n} / \mathbf{Z} . \Delta_{n}^{4}$ to $\mathbf{Z}$. When $n=3$, this is a map from $\mathbf{S L}(2, \mathbf{Z})$ to $\mathbf{Z}$.

We now prove Theorem $\mathrm{B}$ according to which this function reduces to "the" ubiquitous function on $\mathbf{S L}(2, \mathbf{Z})$, that appears in so many different contexts: the Arnold - Atiyah - Brooks - Dedekind - Dupont - EulerGuichardet - Hirzebruch -Kashiwara - Leray- Lion - Maslov - Meyer Rademacher - Souriau - Vergne - Wigner function discussed in $[1,3]$.

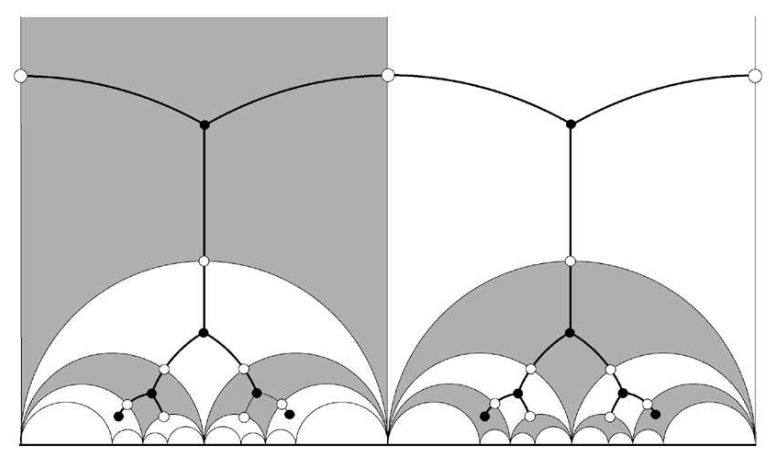

Figure 20: Action of $\mathbf{S L}(2, \mathbf{Z})$ on the tree

Let us recall one of the versions of this function $\Phi$, which is the one used in [5]. The group $\mathbf{S L}(2, \mathbf{Z})$ acts isometrically on Poincaré's upper half space and preserves a trivalent tree whose set of vertices is the orbit of $(1+\sqrt{-3}) / 2$. Given a hyperbolic element $\gamma$ in $\mathbf{S L}(2, \mathbf{Z})$, it preserves a unique embedded line $l_{\gamma}$ in the tree. This line consists of a succession of geodesic arcs in Poincaré's disc and if one moves along this line from a point $x$ to its image $\gamma(x)$, one makes successive left or right turns (with respect to the orientation of Poincaré's half space). Define $\Phi(\gamma)$ to be the number of left turns minus the number of right turns along such a fundamental domain for the action of $\gamma$ on $l_{\gamma}$.

The definition of $\Phi$ on non hyperbolic elements is not so neat (and 
Atiyah's coincidence usually is not valid for such elements). For completeness, we give the values of $\Phi$ on non hyperbolic elements.

Recall that torsion elements in $\mathbf{S L}(2, \mathbf{Z})$ are conjugate of powers of

$$
A=\left(\begin{array}{cc}
0 & 1 \\
-1 & 0
\end{array}\right) \quad\left(=\mathcal{B}_{-1}\left(\sigma_{1} \cdot \sigma_{2} \cdot \sigma_{1}\right)\right)
$$

and

$$
B=\left(\begin{array}{cc}
1 & -1 \\
1 & 0
\end{array}\right) \quad\left(=\mathcal{B}_{-1}\left(\sigma_{1} \cdot \sigma_{2}\right)\right)
$$

of order 4 and 6 respectively. We set $\Phi\left(A^{p}\right)$ to be $0,-3,0,3$ for $p=0,1,2,3$ and $\Phi\left(B^{p}\right)$ to be $0,-4,-2,0,2,4$ for $p=0,1,2,3,4,5$. The action of $A$ (resp. $B$ ) on Poincaré's half space is a rotation of angle $\pi$ (resp. $-2 \pi / 3$ ) around $i=\sqrt{-1}$ (resp. $(1+\sqrt{-3}) / 2)$.

Parabolic elements in $\mathbf{S L}(2, \mathbf{Z})$ are conjugate to powers of

$$
C=\left(\begin{array}{ll}
1 & 1 \\
0 & 1
\end{array}\right) \quad\left(=\mathcal{B}_{-1}\left(\sigma_{2}\right)\right) .
$$

We set $\Phi\left(C^{p}\right)$ to be $p-3$ for $p \geq 1$ and $p+3$ for $p \leq-1$. The remaining non hyperbolic elements in $\mathbf{S L}(2, \mathbf{Z})$ are conjugate to $-C^{p}$. We set $\Phi\left(-C^{p}\right)=-p / 3$.

Theorem 4.2. The function sign $+\frac{2}{3} l k_{3}: \mathbf{S L}(2, \mathbf{Z}) \rightarrow \mathbf{Z}$ coincides with $-1 / 3$ times "the" function $\Phi$.

Proof. The restriction of Meyer cocycle from $\mathbf{S L}(2, \mathbf{R})$ to $\mathbf{S L}(2, \mathbf{Z})$ is exact since $H^{2}(\mathbf{S L}(2, \mathbf{Z}) ; \mathbf{R})=0$. It follows that there is a function $\Psi: \mathbf{S L}(2, \mathbf{Z}) \rightarrow \mathbf{R}$ whose coboundary $d \Psi\left(\gamma_{1}, \gamma_{2}\right)=\Psi\left(\gamma_{1} \gamma_{2}\right)-\Psi\left(\gamma_{1}\right)-\Psi\left(\gamma_{2}\right)$ is $\operatorname{Meyer}\left(\gamma_{1}, \gamma_{2}\right)$. This function $\Psi$ is unique since the difference between two such functions is a homomorphism from $\mathbf{S L}(2, \mathbf{Z})$ to $\mathbf{R}$ and any such homomorphism is trivial $(\mathbf{S L}(2, \mathbf{Z})$ is generated by torsion elements). In [3] this function $\Psi$ is called "the Meyer function" and it is shown to be equal to $1 / 3$ of the Rademacher function $\Phi$. By Theorem A, we know that the coboundary of the function $-\left(\operatorname{sign}+\frac{2}{3} l k_{3}\right)$ is Meyer cocycle. Therefore $-\left(\operatorname{sign}+\frac{2}{3} l k_{3}\right)$ coincides with the Meyer function and hence with $1 / 3$ of Rademacher function. The theorem follows.

Since the proof that the coboundary of $1 / 3$ of the Rademacher function $\Phi$ is Meyer cocycle is somewhat scattered in [3], we believe that it might be useful to reproduce the argument here.

If $x, y$ are two points of the orbit of $i=\sqrt{-1}$ under the action of $\mathbf{S L}(2, \mathbf{Z})$, we denote by $\phi(x, y)$ the number of left turns minus the number of right turns when one moves from $x$ to $y$ in the tree. If $x, y, z$ are three points in the orbit of $i$, one has

$$
\phi(x, y)+\phi(y, z)+\phi(z, x)=-3,0,+3
$$


depending on the "orientation of the triangle $x, y, z$ in the tree". See Figure 21.
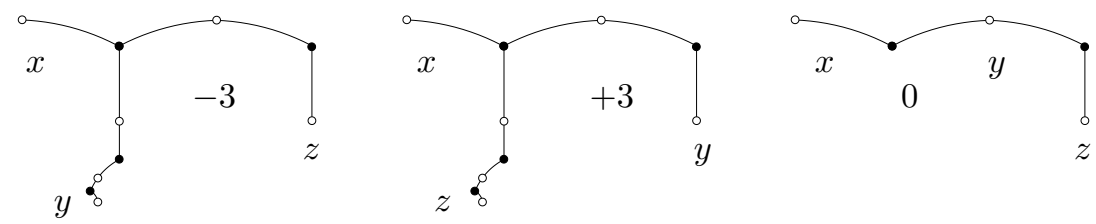

Figure 21: Orientation of a triangle in the tree

Any element $x$ in the orbit of $i$ defines a map

$$
\phi_{x}: \gamma \in \mathbf{S L}(2, \mathbf{Z}) \mapsto \phi(x, \gamma(x)) \in \mathbf{Z}
$$

and it is clear that $\phi_{x}$ and $\phi_{y}$ differ by at most 6 . Moreover, if one chooses $x_{l}=i+l$ (for $l \geq 1$ ), the sequence $\phi_{x_{l}}(\gamma)$ is eventually constant for every $\gamma$ and defines a limit map $\phi_{\infty}: \mathbf{S L}(2, \mathbf{Z}) \rightarrow \mathbf{Z}$ which is also at a bounded distance from the $\phi_{x}$ 's. Note that the definition of the Rademacher function $\Phi$ shows that $\Phi$ and $\phi_{\infty}$ agree on hyperbolic elements and that $\Phi-\phi_{\infty}$ is bounded.

The coboundary $d \phi_{\infty}\left(\gamma_{1}, \gamma_{2}\right)$ is easy to understand. It is equal to $-3,0$ or +3 according to the cyclic ordering of the three points $\left(\infty, \gamma_{1}(\infty), \gamma_{1} \gamma_{2}(\infty)\right)$ in the oriented circle $\mathbf{R} \cup\{\infty\}$ (boundary of Poincaré's half space). It follows that $\frac{1}{3} \phi_{\infty}$ can be extended to $\mathbf{S L}(2, \mathbf{R})$ as the 2-cocycle cycl defined by the same formula. It is not difficult to evaluate the cohomology class of $\mathrm{cycl}$ (for instance by restricting to $\mathbf{S O}(2))$ : one finds that cycl and Meyer are cohomologous cocycles on $\mathbf{S L}(2, \mathbf{R})$. This means that there is a function $u: \mathbf{S L}(2, \mathbf{R}) \rightarrow \mathbf{R}$ such that Meyer - cycl $=d u$. In other words, $u$ is a quasimorphism on $\mathbf{S L}(2, \mathbf{R})$. Any such quasimorphism is bounded since every element of $\mathbf{S L}(2, \mathbf{R})$ can be written as a product of two commutators. Restricting to $\mathbf{S L}(2, \mathbf{Z})$, we find that $\Psi$ and $\frac{1}{3} \phi_{\infty}+u$ differ by a homomorphism and hence coincide. Therefore, we proved that $\left|\Psi-\frac{1}{3} \Phi\right|$ is bounded.

We want to show that $\Psi-\frac{1}{3} \Phi$ vanishes. If $\gamma$ is a hyperbolic element, we know by the definition of the Rademacher function that $\Phi\left(\gamma^{p}\right)=p \Phi(\gamma)$ for every integer $p$. In the same way, we have $\Psi\left(\gamma^{p}\right)=p \Psi(\gamma)$. Indeed,

$$
\Psi\left(\gamma^{p_{1}+p_{2}}\right)-\Psi\left(\gamma^{p_{1}}\right)-\Psi\left(\gamma^{p_{2}}\right)=\operatorname{Meyer}\left(\gamma^{p_{1}}, \gamma^{p_{2}}\right)=0
$$

as we already observed. Hence, if $\Psi-\frac{1}{3} \Phi$ were non trivial on some hyperbolic element, we would conclude that $\Psi-\frac{1}{3} \Phi$ is unbounded on powers of this element, which is a contradiction. The identification of $\Psi$ and $\frac{1}{3} \Phi$ on non hyperbolic elements is just a case by case computation with no interest. Actually, the strange values of $\Phi$ on non hyperbolic elements have been chosen in such a way that this identification holds... 
Let us work out a "numerical example". The braid $\alpha=\sigma_{2}^{3} \cdot \sigma_{1}^{-1} \cdot \sigma_{2} \cdot \sigma_{1}^{-1}$ has a closure which is the mirror image $\overline{6}_{2}$ of the knot $6_{2}$ in Rolfsen's table [12].

Its signature is -2 as one can see in the following way. Write $\alpha=\alpha_{1} \cdot \alpha_{2}$ with $\alpha_{1}=\sigma_{2}^{3} \cdot \sigma_{1}^{-1}$ and $\alpha_{2}=\sigma_{2} \cdot \sigma_{1}^{-1}$. The closure of $\alpha_{2}$ is a trivial braid so that $\operatorname{sign}\left(\alpha_{2}\right)=0$. As for $\hat{\alpha_{1}}$ it coincides with $\hat{\sigma_{2}^{3}}$ whose signature is -2 (as we have seen in Remark 3 in the introduction).

We have by Theorem A

$$
\begin{aligned}
\operatorname{sign}(\hat{\alpha}) & =\operatorname{sign}\left(\hat{\alpha_{1}}\right)+\operatorname{sign}\left(\hat{\alpha_{2}}\right)-\operatorname{Meyer}\left(\mathcal{B}_{-1}\left(\alpha_{1}, \mathcal{B}_{-1}\left(\alpha_{2}\right)\right)\right. \\
& =-2+0 \quad-\operatorname{Meyer}\left(\left(\begin{array}{ll}
4 & 3 \\
1 & 1
\end{array}\right),\left(\begin{array}{ll}
1 & 1 \\
1 & 2
\end{array}\right)\right) \\
& =-2 .
\end{aligned}
$$

On the other hand, since

$$
\mathcal{B}_{-1}\left(\sigma_{1}\right)=A B, \quad \mathcal{B}_{-1}\left(\sigma_{2}\right)=B A
$$

(keeping the notation $A$ and $B$ for the matrices of order 4 and 6 defined above), we have

$$
M=\mathcal{B}_{-1}(\alpha)=(B A)(B A)(B A)\left(B^{-1} A\right)(B A)\left(B^{-1} A\right)=\left(\begin{array}{cc}
7 & 10 \\
2 & 3
\end{array}\right) .
$$
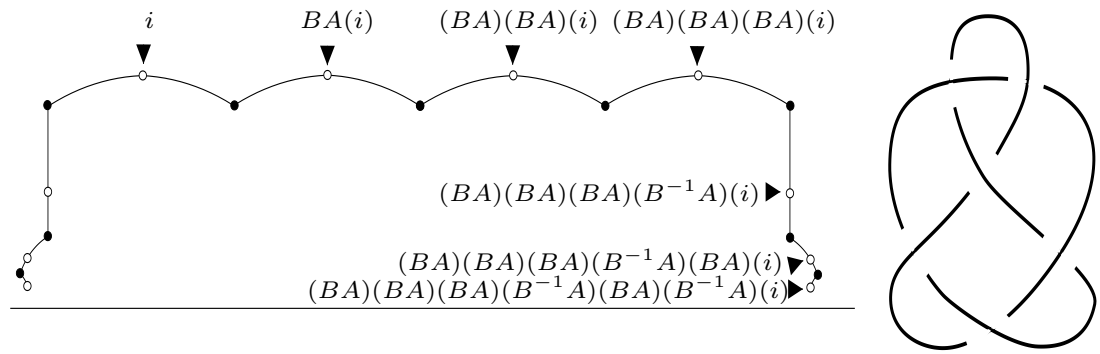

Figure 22: $\Phi\left((B A)(B A)(B A)\left(B^{-1} A\right)(B A)\left(B^{-1} A\right)\right)=2$ and the knot $\overline{6}_{2}$

From this, we see that the line in the tree which is invariant by $M$ has a fundamental domain consisting of 6 pieces, turning successively left, left, left, right, left and right. Hence the Rademacher function $\Phi$ evaluated on $M$ is equal to $4-2=2$ (see Figure 22 ). Since $l k_{3}(\alpha)=2$, we can indeed verify Theorem $\mathrm{A}$ in this case:

$$
-2+\frac{2}{3} \times 2=-\frac{1}{3} \times 2 .
$$


Choose now a primitive $k$-th root of unity $\omega$ and let us compute the $\omega$-signatures of three strands braids.

We know from Section 2 that the image of $\mathcal{B}_{\omega}$ falls into the unitary group of some hermitian form (defined up to sign) which is non degenerate if $\omega$ is not a cubic root of unity. This form is definite (positive or negative) when the real part of $\omega$ is bigger that $-1 / 2$ and has signature $(+,-)$ when this real part is in $[-1,-1 / 2[$.

Assume first that $\Re \omega>1 / 2$. In this case, the Burau-Squier representation $\mathcal{B}_{\omega}$ has an image contained in a group isomorphic to $\mathbf{U}(2) \subset \mathbf{S p}(4, \mathbf{R})$. The restriction of the Meyer cocycle to $\mathbf{U}(n)$ has been made explicit by Souriau, Lion-Vergne and Turaev and we recall the result (in the case of $\mathbf{U}(2)$ ). Define the function $\psi: \mathbf{U}(2) \rightarrow \mathbf{R}$ by

$$
\psi(\gamma)=2\left(\left(\left(\theta_{1}\right)\right)+\left(\left(\theta_{2}\right)\right)\right)
$$

where $\exp \left(2 i \pi \theta_{1}\right)$ and $\exp \left(2 i \pi \theta_{2}\right)$ are the two eigenvalues of $\gamma$ and $(()$.$) :$ $\mathbf{R} \rightarrow \mathbf{R}$ is defined by $((\theta))=\theta-[\theta]-\frac{1}{2}$ if $\theta$ is not an integer ([.] denotes the integral part) and $((\theta))=0$ if $\theta$ is an integer. It turns out that the coboundary of $\psi$ is the restriction of the Meyer cocycle to $\mathbf{U}(2)$ :

$$
\psi\left(\gamma_{1} \gamma_{2}\right)-\psi\left(\gamma_{1}\right)-\psi\left(\gamma_{2}\right)=\operatorname{Meyer}\left(\gamma_{1}, \gamma_{2}\right)
$$

We can now give a formula:

Theorem 4.3. Let $\omega=\exp (2 i \pi \theta)$ be a root of unity such that $0<\theta<1 / 3$. Then the $\omega$-signature of a braid $\alpha$ in $\mathbf{B}_{3}$ is equal to $-2\left(\left(\left(\theta_{1}\right)\right)+\left(\left(\theta_{2}\right)\right)\right)-$ $2 \theta l k_{3}(\alpha)$ where $\exp \left(2 i \pi \theta_{1}\right)$ and $\exp \left(2 i \pi \theta_{2}\right)$ are the two eigenvalues of the matrix $\mathcal{B}_{\omega}(\alpha)$.

Proof. Let $k$ be the order of $\omega$ and $c=\operatorname{sign}_{\omega}\left(\Delta_{3}^{2 k}\right)$. A simple adaptation of Lemma 4.1 shows that $\operatorname{sign}_{\omega}-\frac{c}{6 k} . l k_{3}$ descends to a map from $\mathbf{B}_{3} / \Delta_{3}^{2 k}$ to $\mathbf{Z}$. Its coboundary is given by the opposite of the Meyer cocycle so that both $\operatorname{sign}_{\omega}-\frac{c}{6 k} l k_{3}$ and $-2\left(\left(\left(\theta_{1}\right)\right)+\left(\left(\theta_{2}\right)\right)\right)$ have the same coboundary. The difference, being a homomorphism from $\mathbf{B}_{3} / \Delta_{3}^{2 k}$ to $\mathbf{Z}$, has to vanish. We shall see in Section 5.1 that $c=-12 k \theta$.

Remark that this theorem is not only valid when $n=3$. A similar result holds true, with the same proof, as soon as the hermitian form is definite, i.e. when $\Re \omega>\cos 2 \pi / n$.

Note the following corollary:

Corollary 4.4. Let $\omega=\exp (2 i \pi \theta)$ be a root of unity such that $0<\theta<$ $1 / 3$. Then the $\omega$-signature of a braid $\alpha$ in $\mathbf{B}_{3}$ differs from $-2 \theta l k_{3}(\alpha)$ by at most 2 .

It is not so easy to give a closed formula in the same spirit in case $\Re \omega<-1 / 2$. This is due to the fact that the Maslov class (or Chern 
class) vanishes on $\mathbf{S U}(2)$ (which is simply connected) and does not vanish on $\mathbf{S U}(1,1)$ (which is isomorphic to $\mathbf{P S L}(2, \mathbf{R})$ ). Of course, one can say that $\operatorname{sign}_{\omega}$ can be "easily computed in practice" since it vanishes on the generators $\sigma_{i}$ and we know its coboundary: the Meyer cocycle. This computation is not always so easy... We propose an illustration in the next section.

\section{The gordian space}

\subsection{The $\omega$-signatures of torus knots}

We choose a primitive $k$-th root of unity $\omega$ and an integer $p \geq 1$ and we would like to compute $\operatorname{sign}_{\omega}\left(\sigma_{1} \cdots \sigma_{n-1}\right)^{p}$. When $p$ is a multiple of $n$, this reduces to the computation of the $\omega$-signatures in the center of $\mathbf{B}_{n}$.

Proposition 5.1. Let $\omega=\exp (2 i \pi \theta)$ be a root of unity with $0<\theta<1$. The $\omega$-signature of $\left(\sigma_{1} \cdots \sigma_{n-1}\right)^{p}$ is equal to the Dedekind sum

$$
\operatorname{sign}_{\omega}\left(\sigma_{1} \cdots \sigma_{n-1}\right)^{p}=\sum_{\substack{1 \leq l_{1} \leq n-1 \\ 1 \leq l_{2} \leq p-1}} \epsilon_{\theta}\left[l_{1}, l_{2}\right]
$$

where

$$
\epsilon_{\theta}\left[l_{1}, l_{2}\right]=\left\{\begin{array}{cl}
+1 & \text { if } 0<\theta+l_{1} / n+l_{2} / p<1,(\bmod 2), \\
-1 & \text { if } 1<\theta+l_{1} / n+l_{2} / p<2,(\bmod 2), \\
0 & \text { if } \theta+l_{1} / n+l_{2} / p \in \mathbf{Z} .
\end{array}\right.
$$

Proof. This is a simple adaptation of a proof from [7] page 296, where Kauffman computes the usual signature $(\omega=-1)$ for torus knots. For a suitable Seifert surface for the braid $\left(\sigma_{1} \cdots \sigma_{n-1}\right)^{p}$, the Seifert matrix $B$ is shown to be $-\Lambda_{n-1} \otimes \Lambda_{p-1}$ where $\Lambda_{a}$ denotes the $(a-1) \times(a-1)$ matrix

$$
\left(\begin{array}{ccccc}
1 & -1 & 0 & \ldots & 0 \\
0 & 1 & -1 & \ddots & \vdots \\
\vdots & \ddots & \ddots & \ddots & \vdots \\
\vdots & & \ddots & 1 & -1 \\
0 & \ldots & \ldots & 0 & 1
\end{array}\right) .
$$

Over the complex numbers, $\Lambda_{a}$ can be written as $P_{a} \Omega_{a} P_{a}^{\star}$ where $P_{a}$ is some invertible matrix and

$$
\Omega_{a}=\left(\begin{array}{cccc}
1-\exp (2 i \pi / a) & 0 & \ldots & 0 \\
0 & 1-\exp (2.2 i \pi / a) & & 0 \\
\vdots & \vdots & \ddots & \vdots \\
0 & 0 & \ldots & 1-\exp ((a-1) .2 i \pi / a)
\end{array}\right) .
$$


Therefore, in order to compute the $\omega$-signature of $\left(\sigma_{1} \cdots \sigma_{n-1}\right)^{p}$, one has to compute the signature of the hermitian matrix

$$
-\left[(1-\omega) \Omega_{n} \otimes \Omega_{p}+(1-\bar{\omega})\left(\Omega_{n} \otimes \Omega_{p}\right)^{\star}\right]
$$

which is diagonal. One finds

$$
\operatorname{sign}_{\omega}\left(\sigma_{1} \cdots \sigma_{n-1}\right)^{p}=\sum_{\substack{1 \leq l_{1} \leq n-1 \\ 1 \leq l_{2} \leq p-1}} \operatorname{\sigma ign}\left(\Re(1-\omega)\left(1-\exp \left(2 i \pi l_{1} / n\right)\right)\left(1-\exp \left(2 i \pi l_{2} / p\right)\right)\right)
$$

where бign denotes the sign of a real number (we are out of symbols for signs and signatures!) and $\Re$ the real part of a complex number. Note that

$$
\begin{aligned}
& (1-\omega)\left(1-\exp \left(2 i \pi l_{1} / n\right)\right)\left(1-\exp \left(2 i \pi l_{2} / p\right)\right) \\
& =8 i \sin (\pi \theta) \sin \left(\pi l_{1} / n\right) \sin \left(\pi l_{2} / p\right) \exp \left(i \pi\left(\theta+l_{1} / n+l_{2} / p\right)\right)
\end{aligned}
$$

so that the above formula for the $\omega$-signature reduces to the formula in the proposition. A challenging exercise for the reader would be to give another proof of this proposition using Theorem A.

When $\omega=-1$ and $p=2 n$, one gets the value $1-n^{2}$ that we used earlier.

For every root of unity $\omega$, define the map

$$
\operatorname{Sign}_{\omega}: \alpha \in \mathbf{B}_{n} \mapsto \lim _{p \rightarrow \infty} \frac{1}{p} \operatorname{sign}_{\omega}\left(\alpha^{p}\right) \in \mathbf{R} .
$$

The existence of the limit follows from the quasi-additivity of the sequence $\operatorname{sign}_{\omega}\left(\alpha^{p}\right)$. Note that $\operatorname{Sign}_{\omega}$ is a quasimorphism $\mathbf{B}_{n} \rightarrow \mathbf{R}$ and that the absolute value of the difference between $\operatorname{Sign}_{\omega}$ and $\operatorname{sign}_{\omega}$ is bounded by $2 n$. The interest of this slight modification from $\operatorname{sign}_{\omega}$ to $\operatorname{Sign}_{\omega}$ is that $\operatorname{Sign}_{\omega}$ is homogeneous i.e. $\operatorname{Sign}_{\omega}\left(\alpha^{p}\right)=p \operatorname{Sign}_{\omega}(\alpha)$ for all $p$ and $\alpha$.

As a simple corollary of Proposition 5.1, we give the homogeneous signatures of torus knots. Indeed, fixing $\theta$ and $l_{1}$, the sum $\frac{1}{p} \sum_{1 \leq l_{2} \leq p-1} \epsilon_{\theta}\left[l_{1}, l_{2}\right]$ differs by at most by $1 / p$ from the integral

$$
\int_{0}^{1} \epsilon_{\theta}\left[l_{1}, x\right] d x
$$

where

$$
\epsilon_{\theta}\left[l_{1}, x\right]= \begin{cases}+1 & \text { if } 0<\theta+l_{1} / n+x<1,(\bmod 2), \\ -1 & \text { if } 1<\theta+l_{1} / n+x<2,(\bmod 2) .\end{cases}
$$

It follows that

$$
\operatorname{Sign}_{\exp (2 i \pi \theta)}\left(\sigma_{1} \cdots \sigma_{n-1}\right)=\sum_{1 \leq l_{1} \leq n-1} \int_{0}^{1} \epsilon_{\theta}\left[l_{1}, x\right] d x .
$$

The latter integral is of course easy to determine and we leave the computation to the reader. Here is the result: 
Proposition 5.2. The value of $\operatorname{Sign}_{\exp (2 i \pi \theta)}\left(\sigma_{1} \cdots \sigma_{n-1}\right)$ is a piecewise affine function of $\theta \in[0,1] \cap \mathbf{Q}$. More precisely:

$$
\operatorname{Sign}_{\exp (2 i \pi \theta)}\left(\sigma_{1} \cdots \sigma_{n-1}\right)=-2(n-(2 l-1)) \theta-2 l(l-1) / n
$$

for $(l-1) / n<\theta<l / n,(l=1, \ldots, n)$.

Note in particular that for $0<\theta<1 / n$, one has

$$
\operatorname{Sign}_{\exp (2 i \pi \theta)}\left(\sigma_{1} \cdots \sigma_{n-1}\right)=-2(n-1) \theta
$$

and that when $n$ is odd and $(n-1) / 2 n<\theta<(n+1) / 2 n$

$$
\operatorname{Sign}_{\exp (2 i \pi \theta)}\left(\sigma_{1} \cdots \sigma_{n-1}\right)=\left(1-n^{2}\right) / 2 n .
$$

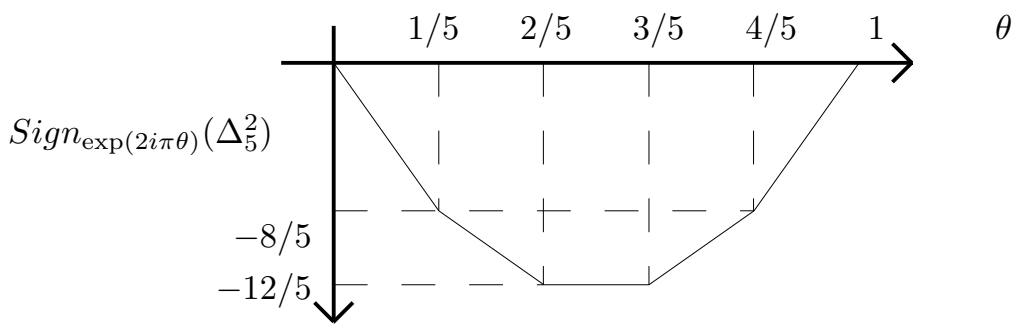

Figure 23: Homogeneous signature

Note also that when $n=3$ and $0<\theta<1 / 3$, we have $\operatorname{Sign}_{\omega}\left(\sigma_{1} \cdot \sigma_{2}\right)=$ $-4 \theta$ so that $\operatorname{sign}_{\omega}\left(\Delta_{3}^{2 k}\right)=\operatorname{sign}_{\omega}\left(\left(\sigma_{1} \cdot \sigma_{2}\right)^{3 k}\right)=-4 \theta .3 k$ if $\omega$ is a $k$-th root of unity. We used this value in the proof of Theorem 4.3.

\subsection{The gordian space}

In this section, we prove Theorem $\mathrm{C}$ according to which it is possible to embed quasi-isometrically copies of $\mathbf{Z}^{d}$ in the gordian metric space $\left(\mathcal{K}\right.$ nots,$\left.d_{\text {gordian }}\right)$. We begin with a simple observation, already made in the case $\omega=-1$ in [15].

Proposition 5.3. For any root of unity $\omega$ and any two knots $\lambda_{1}, \lambda_{2}$, one has

$$
\left|\operatorname{sign}_{\omega}\left(\lambda_{1}\right)-\operatorname{sign}_{\omega}\left(\lambda_{1}\right)\right| \leq 2 d_{\text {gordian }}\left(\lambda_{1}, \lambda_{2}\right) .
$$

Proof. One has to show that if one performs one crossing on a knot then any $\omega$-signature changes by at most 2 . Start with a knot $\lambda$ with a generic projection in the plane and let $\lambda^{\prime}$ be the knot in which one changes one double point of the projection, changing at that point the up and down 
part. There is a classical "Seifert algorithm" (that one finds for instance in [12]) constructing a Seifert surface from a knot projection. Comparing the Seifert matrices for the two knots $\lambda, \lambda^{\prime}$ associated to these Seifert surfaces, one realizes that they only differ by one diagonal term. It follows that the associated quadratic forms agree on a codimension 1 subspace. Therefore, the corresponding signatures differ by at most 2 .

In order to prove the existence of quasi-isometric embeddings of $\mathbf{Z}^{d}$ in the gordian metric space $\mathcal{K}$ nots, we shall need the following lemma.

Lemma 5.4. For each integer $d \geq 1$, one can find $d$ elements $\alpha_{1}, \ldots, \alpha_{d}$ of some braid groups $\mathbf{B}_{n_{1}}, \ldots, \mathbf{B}_{n_{d}}$ and $d$ roots of unity $\omega_{1}, \ldots, \omega_{d}$ such that the $d \times d$ matrix with entries $\operatorname{Sign}_{\omega_{i}}\left(\alpha_{j}\right)$ is non singular.

Let us finish the proof of Theorem $\mathrm{C}$ assuming this lemma.

Denote by $n$ the sum $n_{1}+\ldots+n_{d}$ and consider the braid group $\mathbf{B}_{n}$. Grouping the base points $x_{1}^{0}, \ldots, x_{n}^{0}$ by groups of size $n_{1}, n_{2}, \ldots, n_{d}$, we get $d$ embeddings $\phi_{1}, \ldots, \phi_{d}$ of $\mathbf{B}_{n_{1}}, \ldots, \mathbf{B}_{n_{d}}$ in $\mathbf{B}_{n}$ whose images commute. The choice of $d$ elements $\alpha_{1}, \ldots, \alpha_{d}$ defines a homomorphism

$$
\psi: m=\left(m_{1}, \ldots, m_{d}\right) \in \mathbf{Z}^{d} \mapsto \phi_{1}\left(\alpha_{1}\right)^{m_{1}} \cdots \phi_{d}\left(\alpha_{d}\right)^{m_{d}} \in \mathbf{B}_{n}
$$

Replacing if necessary all the $\alpha_{i}$ 's by some suitable power, we may assume that the image of $\psi$ is contained in the pure braid group, which means that the induced permutation of the $n$ points is trivial. Let $\beta=\sigma_{1} \cdots \sigma_{n-1}$ in $\mathbf{B}_{n}$, which induces a cyclic permutation of the $n$ points, so that any braid of the form $\psi(m) \cdot \beta$ has a closure which is a knot (i.e. which is connected).

We claim that the map

$$
\xi: m \in \mathbf{Z}^{d} \mapsto \widehat{\psi(m) \cdot \beta} \in \mathcal{K} \text { nots }
$$

is a quasi-isometric embedding as required by Theorem C. Recall that we denote with a hat the closure of a braid.

One distance estimate is obvious. If $m_{1}$ and $m_{2}$ are two elements of $\mathbf{Z}^{d}$, the length of the element $\psi\left(m_{1}\right) \psi\left(m_{2}\right)^{-1}$ of $\mathbf{B}_{n}$ in the classical generating system is obviously bounded by some constant times the norm of $m_{1}-m_{2}$. It follows that one can pass from the knot $\widehat{\psi\left(m_{1}\right)} \cdot \beta$ to $\psi \widehat{\left(m_{2}\right)} \cdot \beta$ by "undoing" a number of crossings which is less than a constant times $\left\|m_{1}-m_{2}\right\|$.

The other estimate is of course the interesting one. We have to show that if $m_{1}$ and $m_{2}$ are far apart in $\mathbf{Z}^{d}$, the same is true for the knots $\xi\left(m_{1}\right)$ and $\xi\left(m_{2}\right)$ in $\mathcal{K}$ nots. We shall use Lemma 5.4 and Proposition 5.3. Observe that homogeneous quasimorphisms on abelian groups are homomorphisms (see for instance [3]). This means that the map

$$
\text { Sign }: m \in \mathbf{Z}^{d} \mapsto\left(\operatorname{Sign}_{\omega_{1}}(\psi(m)), \ldots, \operatorname{Sign}_{\omega_{d}}(\psi(m))\right) \in \mathbf{R}^{d}
$$


is a homomorphism which, according to Lemma 5.4, has a discrete cocompact image in $\mathbf{R}^{d}$. Therefore, there is a constant $A>0$ such that for any $m_{1}, m_{2}$

$$
\max _{i=1, \ldots, d}\left|\operatorname{Sign}_{\omega_{i}}\left(\psi\left(m_{1}\right)\right)-\operatorname{Sign}_{\omega_{i}}\left(\psi\left(m_{2}\right)\right)\right| \geq A\left\|m_{1}-m_{2}\right\| .
$$

Now recall that Sign and sign differ by at most $2 n$ on $\mathbf{B}_{n}$, that by Theorem A the signatures of $\psi(m) \cdot \beta$ and $\psi(m)$ differ by at most $2 n$, and that a difference in signatures gives a lower bound for the gordian distance by Proposition 5.3. Therefore, we have finally an estimate

$$
A\left\|m_{1}-m_{2}\right\|-B \leq d_{\text {gordian }}\left(\xi\left(m_{1}\right), \xi\left(m_{2}\right)\right) \leq C\left\|m_{1}-m_{2}\right\|
$$

for some positive constants $A, B, C$. This proves Theorem $\mathrm{C}$.

We still have to prove Lemma 5.4. We choose as the braid $\alpha_{i}$ the element $\sigma_{1} \cdots \sigma_{2 i}$ in $\mathbf{B}_{2 i+1}$ for $i=1, \ldots, d$. We know that $\operatorname{Sign}_{\exp (2 i \pi \theta)}\left(\alpha_{i}\right)$ is a piecewise affine function of $\theta$ which is constant in the interval $[i /(2 i+1),(i+1) /(2 i+1)]$. Choose $\theta_{j}$ as a variable in the interval ] $(j-1) /(2 j-1), j /(2 j+1)\left[\right.$. In the matrix $\operatorname{Sign}_{\exp \left(2 i \pi \theta_{j}\right)}\left(\alpha_{i}\right)$ the entries above the diagonal are constant so that when one expands the determinant, one realizes that it can be written as

$$
\text { a. } \theta_{1} \ldots \theta_{d}+U\left(\theta_{1}, \ldots, \theta_{d}\right)
$$

where $a \neq 0$ and $U$ is a polynomial of degree $\leq d-1$. It follows that this determinant cannot vanish identically and Lemma 5.4 is proved.

Note that the quasi-isometric embedding that we constructed is very explicit and can be made with torus knots.

\section{Added on proofs}

A recent preprint points out an inaccuracy in our paper. It is not true that the reduced Burau representation evaluated at $t=-1$ is additive under disjoint union. However, this very interesting preprint proposes a rather simple way to fix this and to use nevertheless the same trick.

Colored tangles and signatures by David Cimasoni, Anthony Conway http://arxiv.org/abs/1507.07818v2

\section{Bibliography}

[1] AtiYah, M.: The logarithm of the Dedekind $\eta$-function. Math. Ann. 278 (1987), no. 1-4, 335-380.

[2] Atiyah, M. \& Singer, I.M.: The index of elliptic operators. III. Ann. of Math. (2) 87 (1968) 546-604. 
[3] Barge, J. \& Ghys, E.: Cocycles d'Euler et de Maslov. Math. Ann. 294 (1992), no. 2, 235-265.

[4] Birman, J.: Braids, links, and mapping class groups. Annals of Mathematics Studies, 82. Princeton University Press, Princeton, N.J. (1974).

[5] Gambaudo, J.-M. \& Ghys, É.: Commutators and diffeomorphisms of surfaces. To appear in Ergod. Th. \& Dynam. Sys. Special volume in memory of M. Herman (2003).

[6] Goldschmidt, D. M. \& Jones, V.F.R.: Metaplectic link invariants. Geom. Dedicata 31 (1989), no. 2, 165-191.

[7] Kauffman, L.: On knots. Annals of Mathematics Studies 115. Princeton University Press, Princeton, NJ, (1987).

[8] Kolev, B.: Entropie topologique et représentation de Burau. C. $R$. Acad. Sci. Paris Ser. I Math., 309 (13), (1989), 835-838.

[9] Meyer, W.: Die Signatur von Flächenbündeln. Math. Ann., 201 (1973), 239-264.

[10] Murasugi, K.: On closed 3-braids. Memoirs of the American Mathematical Society 151. AMS (1974).

[11] Murasugi, K.: Knot theory and its applications. Translated from the 1993 Japanese original by Bohdan Kurpita. Birkhäuser Boston, Inc., Boston, (1996).

[12] Rolfsen, D.: Knots and links. Mathematics Lecture Series, No. 7. Publish or Perish, Inc., Berkeley, Calif., (1976).

[13] Squier, C. C.: The Burau representation is unitary. Proc. Amer. Math. Soc. 90 (1984), no. 2, 199-202.

[14] Sullivan, D.: On the intersection ring of compact three manifolds. Topology 14 (1975), no. 3, 275-277.

[15] Taniyama, K. \& Yasuhara, A.: On C-distance of knots. Kobe J. Math. 11 (1994), no. 1, 117-127.

[16] Turaev, V.G.: A cocycle of the symplectic first Chern class and Maslov indices. (Russian) Funktsional. Anal. i Prilozhen. 18 (1984), no. 1, 43-48. English translation: Functional Anal. Appl. 18 (1984), no. $1,35-39$.

[17] Wall, C.T.C.: Non-additivity of the signature. Invent. Math. 7 (1969) 269-274. 
Jean-Marc Gambaudo

Institut Non Linéaire de Nice - Sophia Antipolis U.M.R. CNRS 7335

Université de Nice - Sophia - Antipolis 1361, route des Lucioles 06560, Valbonne France.

jean-marc.gambaudo@inln.cnrs.fr

http://www.inln.cnrs.fr/personnel/gambaudo

Étienne Ghys

Unité de Mathématiques Pures et Appliquées de l'École Normale Supérieure de Lyon U.M.R. 5669 du CNRS

46, Allée d'Italie 69364 Lyon Cedex 07- France

etienne.ghys@ens-lyon.fr

http://perso.ens-lyon.fr/ghys/ 